\title{
Investigation of the Turbulent Structure of a Cloud-Capped Mixed Layer Using Doppler Radar
}

\author{
M. PINSKY \\ The Hebrew University of Jerusalem, Jerusalem, Israel \\ O. KRASNOV AND H. W. J. RUSSCHENBERG \\ International Research Centre for Telecommunications-transmission and Radar, Faculty of Information Technology and
} Systems, Delft University of Technology, Delft, Netherlands

A. KHAIN

The Hebrew University of Jerusalem, Jerusalem, Israel

(Manuscript received 11 May 2009, in final form 11 January 2010)

\begin{abstract}
A new method for retrieving air velocity fluctuations in the cloud-capped boundary layer (BL) using radar reflectivity and the Doppler velocity fields is proposed. The method was developed on the basis of data obtained by the Transportable Atmospheric Radar (TARA) located in Cabauw, Netherlands, at 05000812 UTC 8 May 2004, and tested using a detailed trajectory ensemble model of the cloud-capped BL. During the observations, the BL depth was $1200 \mathrm{~m}$, and the cloud base (measured by a lidar) was at $500-550 \mathrm{~m}$. No preliminary assumptions concerning the shapes of drop size distributions were made. On the basis of the TARA radar data, vertical profiles of the vertical air velocity standard deviation, of turbulent dissipation rate, etc. were estimated. The correlation functions indicate the existence of large eddies in the BL with a characteristic horizontal scale of about $600 \mathrm{~m}$. Analysis of the slope (the scaling parameter) of the structure functions indicates that turbulence above $400 \mathrm{~m}$ can be considered to be isotropic. Below this level, the turbulence becomes anisotropic. The rate of anisotropy increases with the decrease of the height above the surface. The averaged values of the dissipation rate were evaluated as $1-2 \mathrm{~cm}^{2} \mathrm{~s}^{-3}$. The importance of using the cloud-capped BL model as a link between different types of observed data (radar, lidar, aircraft, etc.) is discussed. More data should be analyzed to understand the changes in the turbulent structure of the BL during its growth, as well as during cloud and drizzle formation.
\end{abstract}

\section{Introduction}

For decades, the turbulent structure of the atmospheric boundary layer $(\mathrm{ABL})$ has been the focus of many studies (e.g., LeMone 1973, 1990; Lenschow et al. 1980; Bizova et al. 1989; Duynkerke et al. 1995; Lothon et al. 2005; Kollias and Albrecht 2000; Kollias et al. 2001; Yanovsky et al. 2005; Siebert et al. 2006). Investigation into the turbulent structure of the cloud-capped BL is of special interest because of the mutual influence between cloud microphysics and boundary layer dynamics. For instance,

Corresponding author address: Prof. Alexander Khain, Dept. of the Atmospheric Sciences, The Hebrew University of Jerusalem, Givat Ram 91904, Israel.

E-mail:khain@vms.huji.ac.il
Stevens et al. (2005a,b) and Petters et al. (2006) show that drizzle formation is often accompanied by the formation of open cells within the initially uniform stratocumulus clouds. Vertical velocity fluctuations affect the fluctuation of supersaturation and influence drop concentration. The turbulent structure of the BL determines the rate of turbulent mixing within the BL as well as the rate of dryair entrainment from above.

A vertically pointed Doppler radar is an efficient tool for investigating cloud microphysical parameters, as well as the dynamical parameters of the ABL (e.g., Frisch et al. 1995; Babb and Verlinde 1999; Yanovsky et al. 2005; Gage et al. 1999; Moisseev et al. 2006). Two main fields are usually measured by a vertically pointed Doppler radar, namely, the radar reflectivity $Z(h, t)$ and the mean vertical velocity $V(h, t)$ (the first moment of the Doppler 
spectrum). The vertical velocity $V(h, t)$, measured by a vertically pointed Doppler radar, represents the sum of the air velocity $W(h, t)$ and the sedimentation velocity of cloud and drizzle drops, weighted by the reflectivity of drops, $V_{g}(h, t)$ :

$$
V(h, t)=W(h, t)+V_{g}(h, t),
$$

where $h$ is the vertical coordinate (height), $t$ is the time, and

$$
V_{g}=\frac{1}{Z} \int_{0}^{\infty} r^{6} N(r) V_{d}(r) d r
$$

where $r$ is the drop radius, $N(r)$ is the drop size distribution (hereinafter referred to as DSD), and $V_{d}(r)$ is the sedimentation velocity of drops with radius $r$. The reflectivity $Z$ is defined as

$$
Z=\int_{0}^{\infty} r^{6} N(r) d r
$$

The values of $V(h, t)$ measured by a Doppler radar have a limited range of applicability. In fact, the values $W(h, t)$ and $V_{g}(h, t)$ are often of the same order of magnitude, so, as a rule, it is difficult to attribute $V(h, t)$ directly to the effects of drop sedimentation or to turbulent velocity fluctuations. To investigate the turbulent structure of the BL, as well as cloud microphysical properties, $W(h, t)$ and $V_{g}(h, t)$ in Eq. (1) should be separated. There are several methods for performing such a separation (e.g., Babb and Verlinde 1999; Yanovsky et al. 2005; Lothon et al. 2005; Shupe 2008). Many existing retrieval methods are based on the assumption that the DSDs have a specific shape, given as the gamma or the Marshal-Palmer distributions with several parameters fixed (e.g., Campistron et al. 1991; Orr and Kropfli 1999; Yanovsky et al. 2005; O'Connor et al. 2005). In reality, the DSD shapes in stratocumulus clouds (as well as in cumulus clouds) vary dramatically across space and time (e.g., Korolev 1995; Pinsky et al. 2008; Magaritz et al. 2009) and depend on the stage of cloud evolution. In many cases, clouds contain small droplets, as well as large drizzle drops. The DSD dispersion, which is determined as the ratio of the DSD width to the mean radius, varies within a cloud from 0.05 to 1 at distances of a few tens of meters.

The techniques developed by Shupe et al. (2008) rely on the accurate separation of different modalities in the Doppler velocity spectrum. However, in the case of drizzle (especially a weak drizzle) in stratocumulus, the difference between the spectrum of the vertical air velocity and that of the particle sedimentation velocity is often too low to be discernable with radar. Lothon et al. (2005) divided the measured Doppler velocity into the air velocity and the drop sedimentation velocity using the available simultaneous DSD measurements of airborne probes. However, in situ measured DSD are usually available only in specific field campaigns.

Thus, a retrieval method is required that is not based on the spectrum separation and does not use additional information regarding the DSD. In the present paper, an algorithm of such a method is presented and tested using the results of a trajectory ensemble model of the cloudcapped BL (Pinsky et al. 2008). This method is applied to an analysis of the turbulent structure of the cloudcapped mixed layer.

\section{Statistical properties of the Doppler velocity field and their interpretation}

The proposed method is illustrated using the radar reflectivity and the Doppler velocity fields, measured on 8 May 2004 during the time period 0500-0812 UTC with the S-band Transportable Atmospheric Radar (TARA) located in Cabauw, Netherlands. The measurement results are shown in Fig. 1. The radar measured the mean Doppler velocity every $0.5 \mathrm{~s}$, with a radial resolution of $30 \mathrm{~m}$ by integration of the full Doppler velocity spectrum. The beamwidth of the radar antenna was $1.5^{\circ}$. The sensitivity at $5 \mathrm{~km}$ equaled $-30 \mathrm{dBZ}$ [more information about TARA can be found in Heijnen et al. (2000)]. As one can see in Fig. 1, the initially very thin cloud layer, with a reflectivity of $-10 \mathrm{~dB} Z$, develops and starts drizzling, so that the radar reflectivity increases to $10 \mathrm{dBZ}$. This reflectivity corresponds to a comparatively weak drizzle (Stevens et al. 2003, 2005a). One can see that the radar reflectivity from the drizzle decreases to $-10 \mathrm{~dB} Z$ toward the surface within the lower 200-m layer, which can be attributed to evaporation of drizzle within a comparatively dry air. During the preset period, the surface temperature was about $12^{\circ} \mathrm{C}$. As a drizzling cloud was developing, a well-mixed layer formed, with a virtual potential temperature of $285 \mathrm{~K}$. During the drizzle period, the cloud base was at $500-550 \mathrm{~m}$ and the cloud top at $1200 \mathrm{~m}$. The mean horizontal wind velocity within the lower $1.5-\mathrm{km}$ layer increased with height from 6 to $9 \mathrm{~m} \mathrm{~s}^{-1}$, while changes in wind direction with height were about $10^{\circ}$, which corresponds to a change in the wind speed of about $10 \%-15 \%$ of the background wind speed. The white band seen in Fig. 1 is caused by a technical problem in the measurements.

The proposed method is illustrated and tested using the novel trajectory ensemble model of a cloud-capped BL (Pinsky et al. 2008; Khain et al. 2008; Magaritz et al. 2009). A brief description of the model is presented in appendix A. The main specific feature of the model is an accurate reproduction of the spatial variations of 


\section{Reflectivity, dBZ}

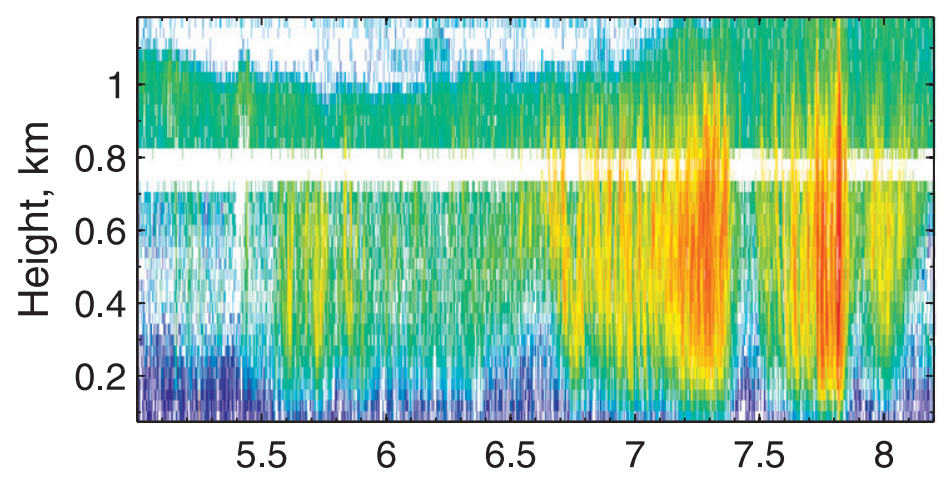

Vertical velocity, $\mathrm{m} / \mathrm{s}$

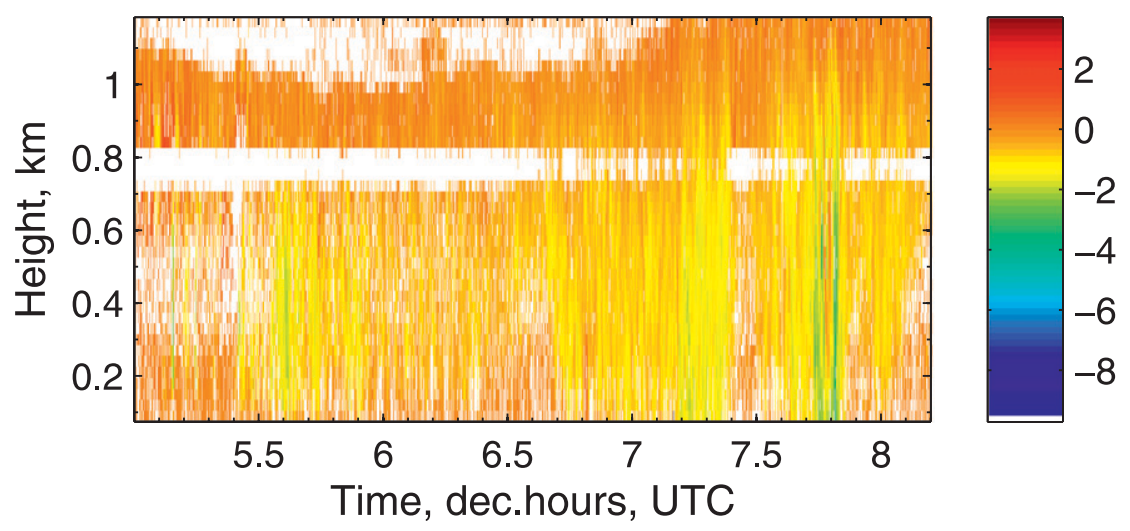

FIG. 1. Time series of the height profiles of the (top) radar reflectivity $Z(h, t)$ and (bottom) vertical Doppler velocity $V(h, t)$ measured by the TARA radar in Cabauw on 8 May 2004. the drop concentration, of the DSD shapes, and of the gravitational drop sedimentation. A good level of agreement was obtained between the dynamical and microphysical structures simulated by the model (including droplet concentration, liquid water content, radar reflectivity, and drizzle fluxes) and the observations made in the second Dynamics and Chemistry of Marine Stratocumulus (DYCOMS-II) field experiment.

The model calculates the air velocity and DSD at each point of the BL. These values allow for the calculation of the Doppler velocity field. To mimic the measurements of a vertically pointed Doppler radar, the turbulent-like velocity field and drops were advected horizontally by a background flow with velocity of $10 \mathrm{~m} \mathrm{~s}^{-1}$. The timeheight series of the Doppler velocity field, with time and height resolutions equal to $1 \mathrm{~s}$ and $30 \mathrm{~m}$, respectively, was calculated with the help of the frozen turbulence hypothesis in order to simulate velocities measured by a vertically pointed Doppler radar. The model-simulated Doppler spectrum represents the sum of the mean turbulent velocity in a given parcel and the distribution of drop-settling velocities weighted by the reflectivity. The time-height series of the radar reflectivity and the Doppler velocity calculated by the model were used as the input for testing the retrieval method. It was assumed that in case the method is accurate, it should restore air velocities and drop sedimentation velocities calculated by the model.

The model was applied to simulate the maritime BL observed during the DYCOMS II field experiment (research flight RF07). This particular case was chosen due to the availability of well-documented microphysical and dynamical data. The cloud base and cloud top were at 400 and $840 \mathrm{~m}$, respectively. The area-averaged mixing ratio was about $10 \mathrm{~g} \mathrm{~kg}^{-1}$ the droplet concentration was $\sim 160 \mathrm{~cm}^{-3}$. Detailed descriptions of the ambient conditions, as well as a comparison between the model results and the in situ measurements, are presented in Pinsky et al. (2008) and Magaritz et al. (2009). The model dynamics were adapted to the observations; for instance, adaptations were made in the structure function (studied by Lothon et al. 2005) and in the vertical profiles of the standard deviation of the vertical velocity fluctuations. Size distributions of aerosols as well as of drops were 
measured in situ. The simulation performed on the data of this case study allowed for a detailed comparison of DSDs and drizzle flux data obtained from simulations with those obtained from observations. The Doppler velocity field and radar reflectivity were calculated by the model using the model output.

In fact, utilization of the model allowed us to perform an ideal observing system simulation experiment, since no measurement noise was introduced into the experiment.

None of the dynamical and microphysical data were available in the Cabauw experiment. At the same time, the maximum radar reflectivity in RF07 was $\sim 2 \mathrm{dBZ}$, indicating that in RF07 the observed drizzle was weak, but stronger than in the Cabauw case. Since we suppose that the retrieval method proposed is suitable for cases of weak drizzle, it should work in both cases. Some thermodynamical conditions (e.g., surface temperatures) were different in RF07 than in the Cabauw case. Applying the method to these two cases of weak drizzle justifies its applicability under different conditions.

Before introducing the method for separating the two Doppler velocity components, we will consider some statistical properties and relationships of the Doppler velocity field and the reflectivity field. Statistical moments are calculated using the Cabauw observation data and are illustrated based on the results of the model simulation.

\section{a. Mean profiles and $V-Z$ scattering diagram}

Figures $2 \mathrm{a}$ and $2 \mathrm{~b}$ show the $V-Z$ scattering diagrams obtained from the observations using the TARA Doppler radar (Cabauw) and the data simulated by the model for maritime stratocumulus. Both panels in Fig. 2 show that at low $Z$ the dependence of $V$ on $Z$ is more or less symmetrical with respect to the horizontal line (around zero velocity), which indicates the low contribution of the drop sedimentation to the total Doppler velocity. However, at higher values of $Z, V$ becomes negative (directed downward), with the absolute value increasing with the increase in $Z$. This tendency indicates the increasing contribution of the drop sedimentation velocity to the Doppler radar velocity.

Analysis of the mean vertical profiles averaged over the time period chosen (192 min; see Fig. 3) shows that the maximum values of radar reflectivity in both cases take place at $\mathrm{h} \sim 500-550 \mathrm{~m}$, which corresponds to the cloud-base level. (The peak at $\sim 800 \mathrm{~m}$ in the Cabauw case is attributed to a technical problem in the measurements and corresponds to the white line seen in Fig. 1.) The largest values in radar reflectivity are around $-7 \mathrm{dBZ}$ in the Cabauw case and $-2 \mathrm{~dB} Z$ in the simulated maritime cloud. Therefore, the drizzle drops are larger in the DYCOMS-II case. In the Cabauw case, small drizzle largely evaporates toward the surface (the radar reflectivity decreases to $-30 \mathrm{dBZ}$ ), while in the DYCOMS II case the drizzle falls on the surface because of larger drop sizes and higher relative humidity over the ocean. The averaged value of the Doppler velocity $\langle V\rangle$ has a negative value, increasing toward the earth's surface. Close to the surface, the fluctuations of the Doppler velocity are not equal to zero. Note that in spite of the fact that most drizzle evaporates in the Cabauw case, a few of the largest drizzle drops remain, causing the relatively large values of the Doppler velocity, $0.5 \mathrm{~m} \mathrm{~s}^{-1}$ at the surface. Up to 100-m height, the signal strength also diminishes as a result of the antenna's near-field effects. This has a limited effect on the estimated vertical velocity.

\section{b. Mean relationships between the Doppler velocity and reflectivity fields}

To understand the nature of the Doppler velocity field, we divided the total range of the radar reflectivity into intervals with a $0.25-\mathrm{dBZ}$ step and calculated the mean velocity and the velocity standard deviation for each interval. Figure 4 shows the results of the calculation of these statistical quantities obtained from the TARA Doppler radar observations (left) and the quantities simulated by the model for maritime stratocumulus (right). The vertical velocity is averaged over time (which corresponds to the averaging in the horizontal direction). This averaging was carried out over all reflectivity classes.

One can see that the averaged negative deviation of the velocity increases with the increase in reflectivity. It is well known that horizontally averaged vertical air velocity $\langle W(h)\rangle$ in the BL is very close to zero (Stevens et al. 2003):

$$
\langle W(h)\rangle=\frac{1}{N} \sum_{k=1}^{N} W\left(h, t_{k}\right)=0 .
$$

Hence, this negative deviation of the Doppler velocity, increasing with the increase in radar reflectivity, is related to drop sedimentation. The dependence shown in Fig. 4 actually represents the average dependence between the sedimentation velocity and the reflectivity.

Figure 4 also indicates the dependence of the velocity standard deviation on reflectivity. However, it is reasonable to suggest that air velocity fluctuations (at least in cases of no or weak drizzle) are not related to the reflectivity. Hence, the dependence can be attributed to the spatial and temporal variances of the sedimentation velocity corresponding to the DSD shape variance. Usually, the minimal velocity standard deviation is observed at small values of reflectivity (see Fig. 4a), when drops are small and move with air, which means that the Doppler velocity is determined by the air velocity. In some cases (Fig. 4c), however, the minimal velocity standard deviation can be found at reflectivities of between -10 and $-20 \mathrm{dBZ}$. 
(a)

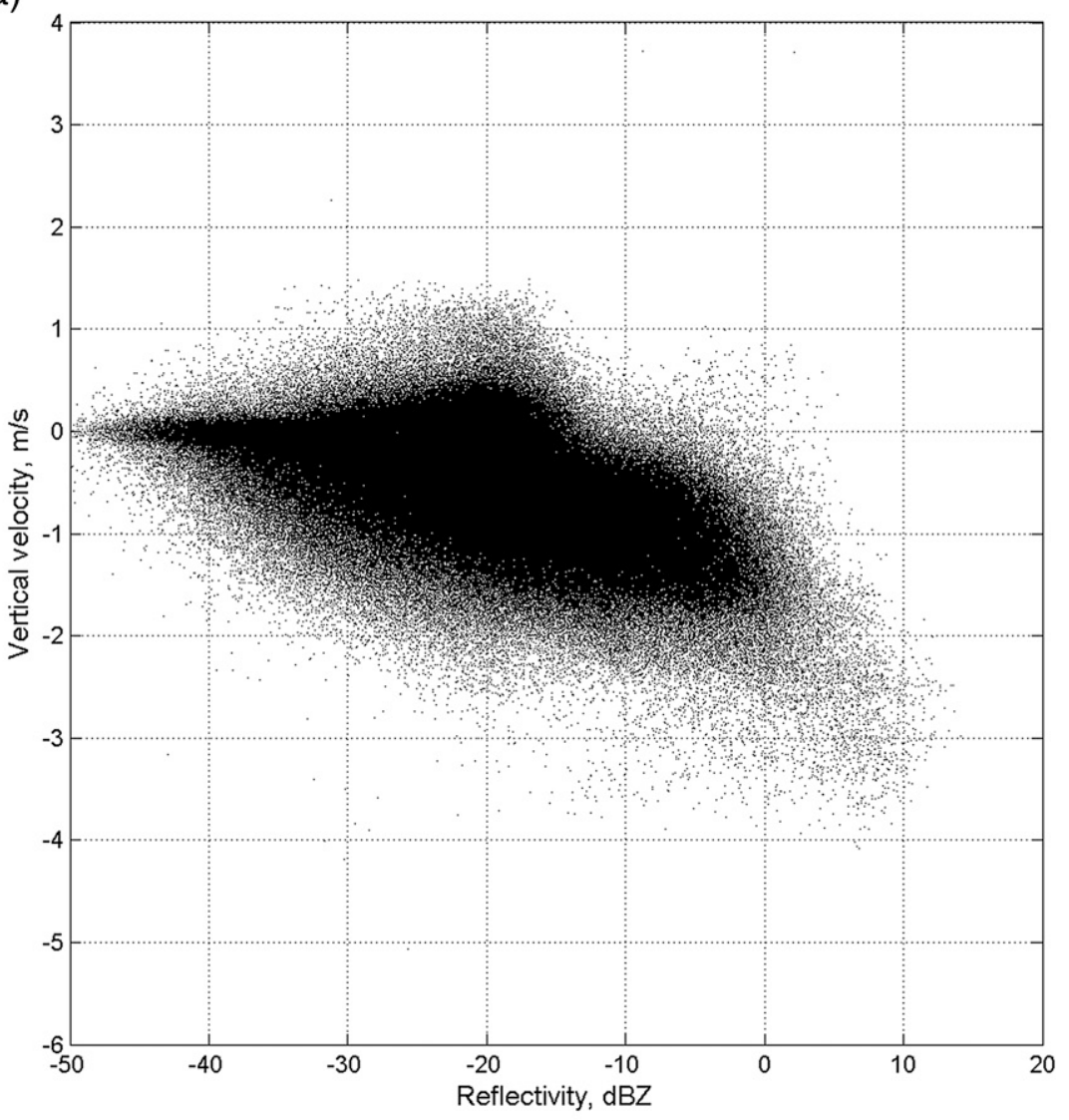

(b)

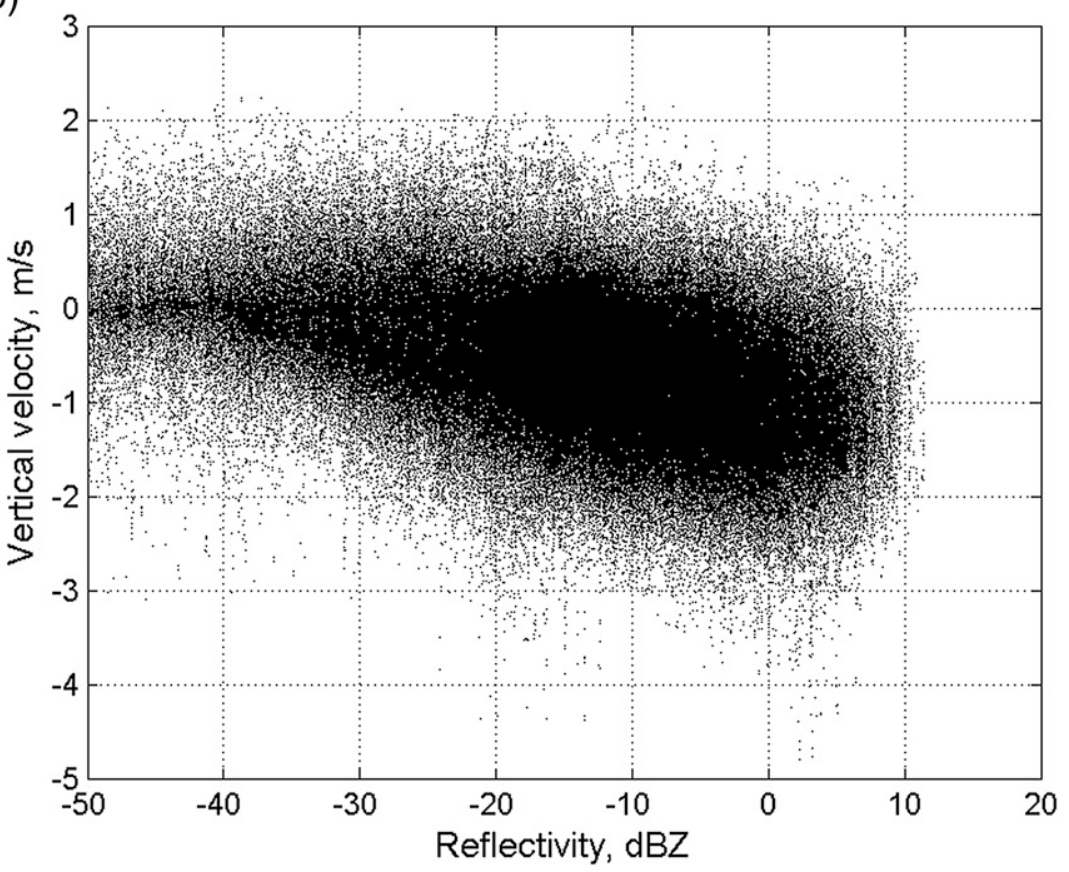

FIG. 2. The $V-Z$ scattering diagrams (a) obtained during observations using the TARA Doppler radar and (b) simulated by the TEM model for maritime stratocumulus. Dots denote the corresponding values measured (calculated) within the layer of 30-m depth with a time increment of $0.5 \mathrm{~s}$ for the observations and $1 \mathrm{~s}$ for the model. 
(a)
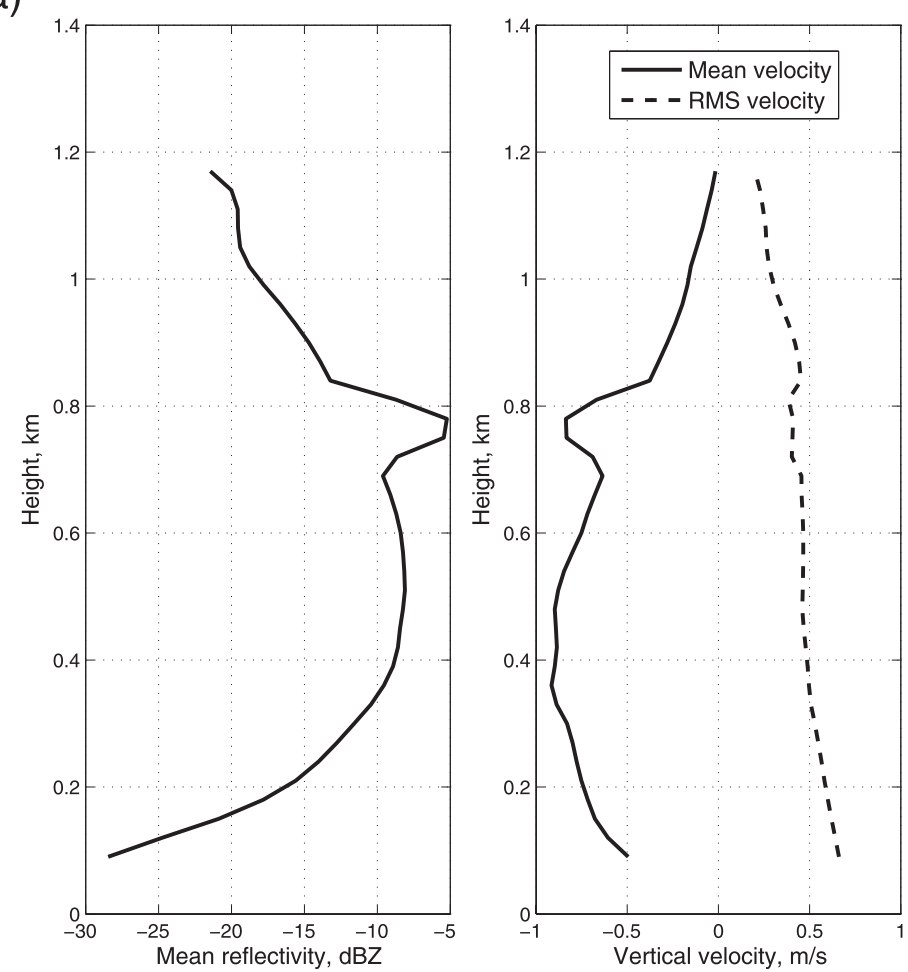

(b)
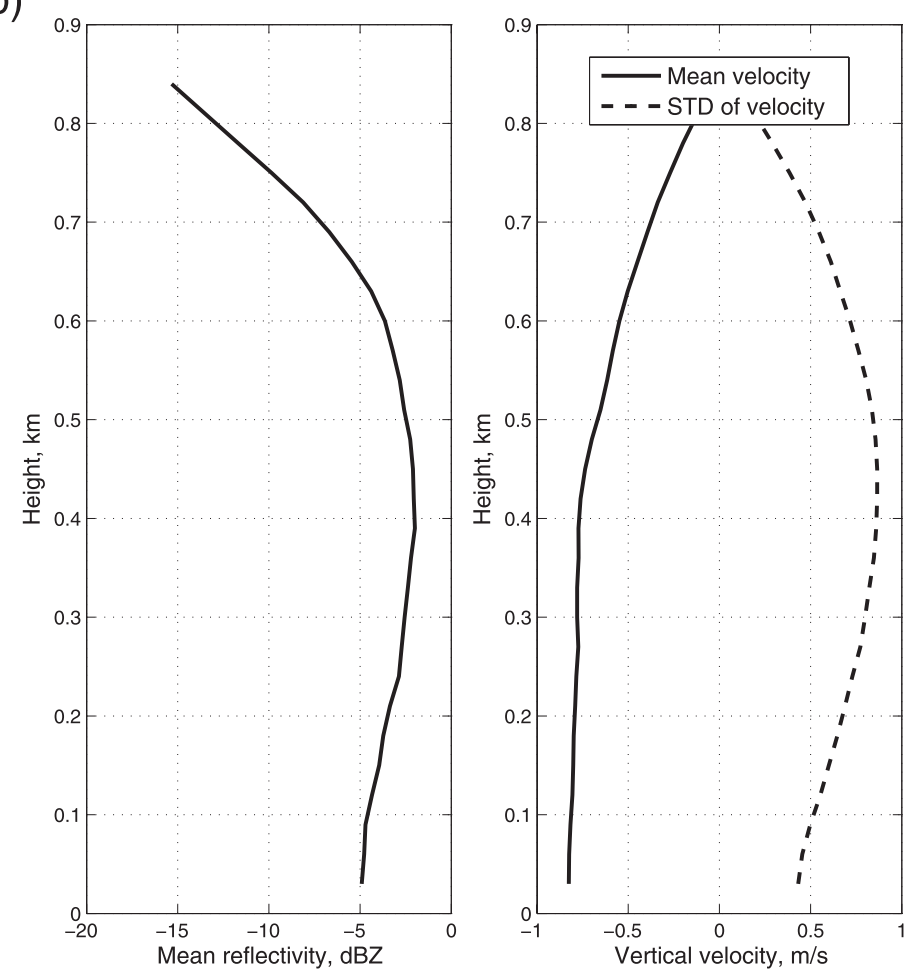

FIG. 3. Vertical profiles of the (left) mean radar reflectivity and (right) the mean Doppler velocity and its RMS that were (a) obtained from observations using the TARA Doppler radar and (b) simulated by the TEM model for maritime stratocumulus. 
(a)

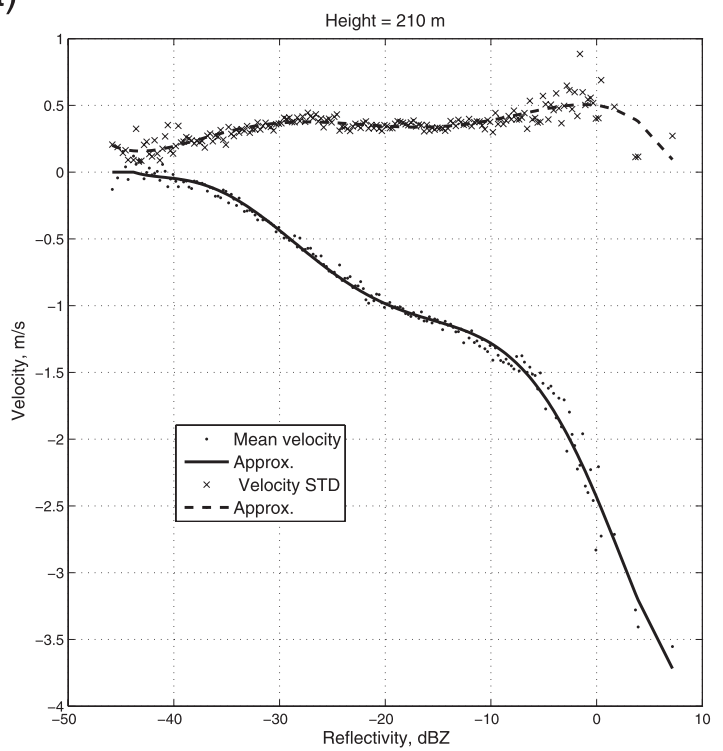

(c)

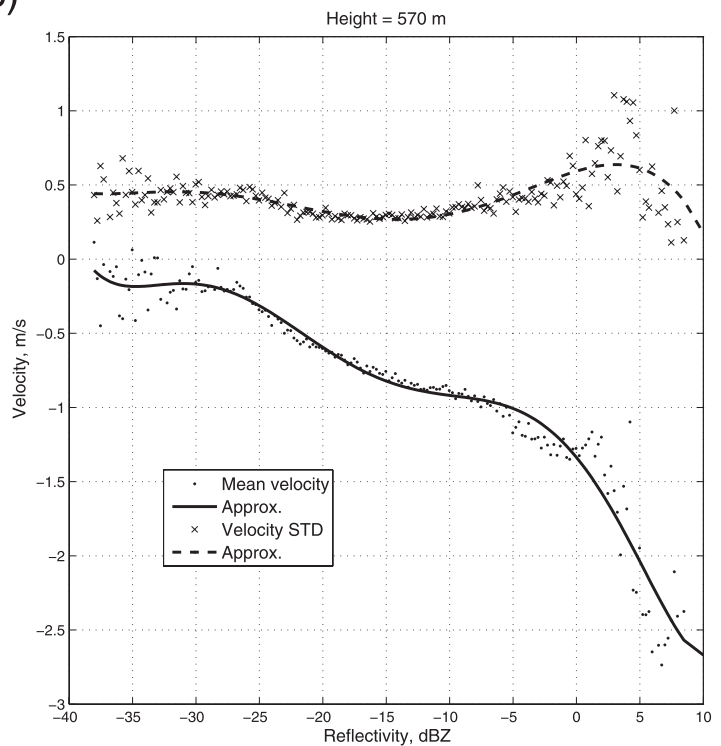

(b)

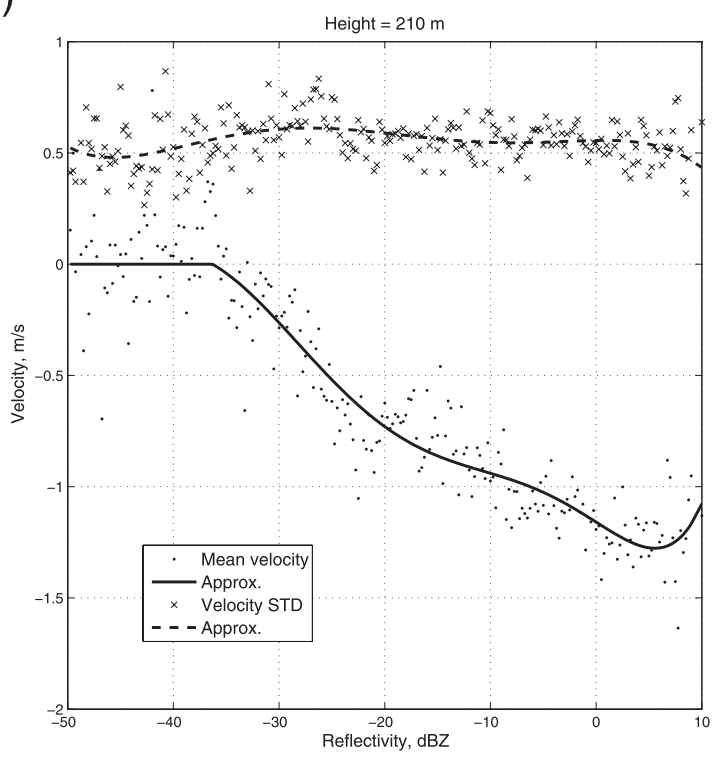

(d)

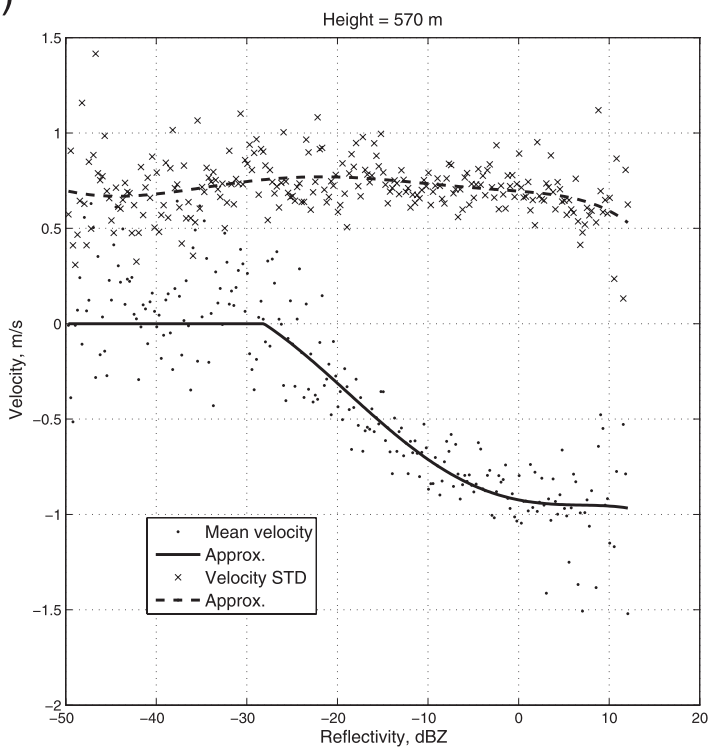

FIG. 4. The mean Doppler velocity and its RMS as a function of the radar reflectivities at (top) 210 and (bottom) $570 \mathrm{~m}$ : (a),(c) obtained from observations using the TARA Doppler radar and (b),(d) simulated by the model for maritime stratocumulus. The solid lines are a polynomial approximation of the data.

In this case, the Doppler signals are formed both by small cloud droplets and by a small fraction of the relatively large drizzle drops.

Note that Figs. $4 \mathrm{~b}$ and $4 \mathrm{~d}$ show higher scattering in the diagrams relating to the model calculations. We attribute this result to the fact that while the Cabauw data are the result of averaging over the radar volume, such averaging was not performed in the model simulations. Estimations show that this can lead to errors of $2-3 \mathrm{~cm} \mathrm{~s}^{-1}$ in the estimation of the mean velocity profiles in the model calculations.

\section{c. Correlation between the fields of the reflectivity, sedimentation velocity, and air velocity}

The formation of the DSD in stratocumulus clouds is a complex process depending on both dynamical and microphysical structures of a cloud. In the cases of no or weak drizzle, one can expect only a weak correlation between DSD moments and air velocity fluctuations. We checked this hypothesis using the model data. Figure 5 shows vertical profiles of the coefficient of correlation between the reflectivity and air velocity fluctuations, as 


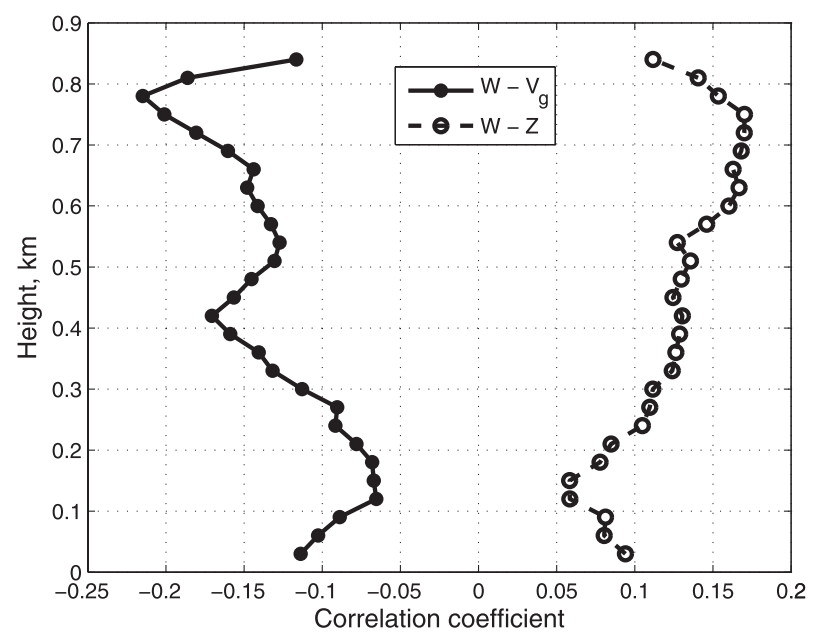

FIG. 5. Vertical profiles of the correlation coefficients between the reflectivity and air velocity fluctuation (left profile) and the sedimentation and air velocity fluctuation (right profile).

well as between the sedimentation and air velocity fluctuations. The absolute values of the correlation coefficient do not exceed 0.2, which means the correlation is quite low. The increase in the correlation over height seen in Fig. 5 can be explained by the fact that droplets grow with height in cloud updrafts and reach their largest size near the cloud top. Note that the model does not take into account the effects of drop evaporation below cloud base on the vertical air velocities. However, we believe that for cases of no or weak drizzle such effect should be quite small. Therefore, hereinafter, we assume zero correlations:

$$
\begin{gathered}
\left\langle W(h) Z^{\prime}(h)\right\rangle=\frac{1}{N} \sum_{k=1}^{N} W\left(h, t_{k}\right) Z^{\prime}\left(h, t_{k}\right)=0 \quad \text { and } \\
\left\langle W(h) V_{g}^{\prime}(h)\right\rangle=\frac{1}{N} \sum_{k=1}^{N} W\left(h, t_{k}\right) V_{g}^{\prime}\left(h, t_{k}\right)=0,
\end{gathered}
$$

where $Z^{\prime}\left(h, t_{k}\right)$ and $V_{g}^{\prime}\left(h, t_{k}\right)$ are deviations from the corresponding mean values.

The variation of the Doppler velocity in this case can be written as

$$
\begin{aligned}
\left\langle[V(h)-\langle V(h)\rangle]^{2}\right\rangle & =\left\langle W^{2}(h)\right\rangle+\left\langle\left[V_{g}-\left\langle V_{g}(h)\right\rangle\right]^{2}\right\rangle \\
& =\left\langle W^{2}(h)\right\rangle+\left\langle V_{g}^{\prime 2}(h)\right\rangle .
\end{aligned}
$$

We suppose that these assumptions are valid for stratocumulus clouds, where drop loading is small and can hardly affect the air velocity field.

\section{d. Errors in Doppler velocity measurements seen in the first lags of the structure function}

Independent errors usually occur in measurements of the Doppler velocity in any reflective volume. The
TARA resolution is $8.9 \mathrm{~cm} \mathrm{~s}^{-1}$. In real measurements, the errors in the Doppler velocity measurements depend on different factors (signal-noise ratio, the Doppler spectrum width, etc.). We estimate these errors as follows. Figure 6a shows several first lags of the Doppler velocity structure function, $D(\tau)$. One can see a sharp spike at the first lag. The shape of this structure function is typical of the sum of the correlated random signal and white noise (errors). In a case where the time lag $\tau_{0}$ is much smaller than the characteristic correlation time, the magnitude of the spike can be used for evaluation of the RMS errors. If the Doppler velocity is measured with a random white error $\eta$, the first lag of the structure function is determined by the variance of $\eta, D\left(\tau_{0}\right) \approx$ $2\left\langle\eta^{2}\right\rangle$, and the RMS error can be estimated as

$$
\sigma_{\eta}=\sqrt{\eta^{2}}=\sqrt{D\left(\tau_{0}\right) / 2}
$$

Figure $6 \mathrm{~b}$ shows the profile of the RMS errors of the Doppler velocity. This error does not influence the mean velocity estimation, but leads to an overestimation of the velocity variance. This profile will be used to correct the estimations of the velocity field moments.

\section{Separation technique}

The main idea of the suggested method is to divide the Doppler velocity field into its air velocity, $\hat{W}(h, t)$, and sedimentation velocity, $\hat{V}_{g}(h, t)$, components in such a way that their sum would obey the statistical properties of the measured Doppler velocity field. Hypotheses (4) and (5) will be used as a priori information. The proposed algorithm of air velocity estimation consists of two steps. In the first step, we exclude the mean negative deviation of the measured Doppler velocity (seen in Figs. 2-4) that is attributed to the drop sedimentation velocity. In the second step, we exclude the standard deviation variations of the Doppler velocity (see Fig. 4), which we also attribute to the effects of drops (caused by droplet sedimentation and DSD variance) on the Doppler velocity.

\section{a. Horizontally averaged fields}

The mean negative deviation of the Doppler velocity strongly correlates with the radar reflectivity because both the reflectivity and the sedimentation velocity increase with drop size. As a result, for each height level it is possible to define a relationship, averaged in the horizontal direction, between the radar reflectivity, $\langle Z\rangle$, and the sedimentation velocity, $\langle V\rangle=\left\langle V_{g}\right\rangle=\varphi(\langle Z\rangle)$. Since at the smallest and the largest values of the reflectivity there cannot be enough statistics available, we cannot immediately use the dependence shown in Fig. 4 by dots. Instead, we use the least mean square approximation of 
(a)

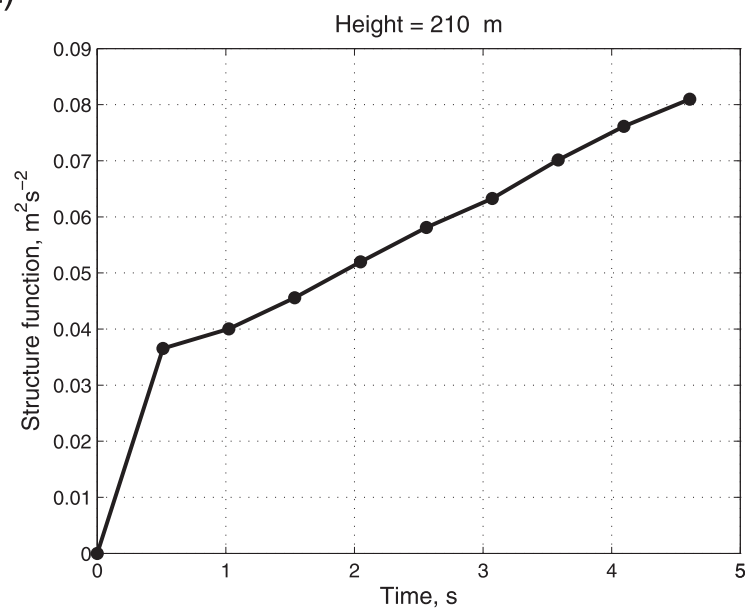

(b)

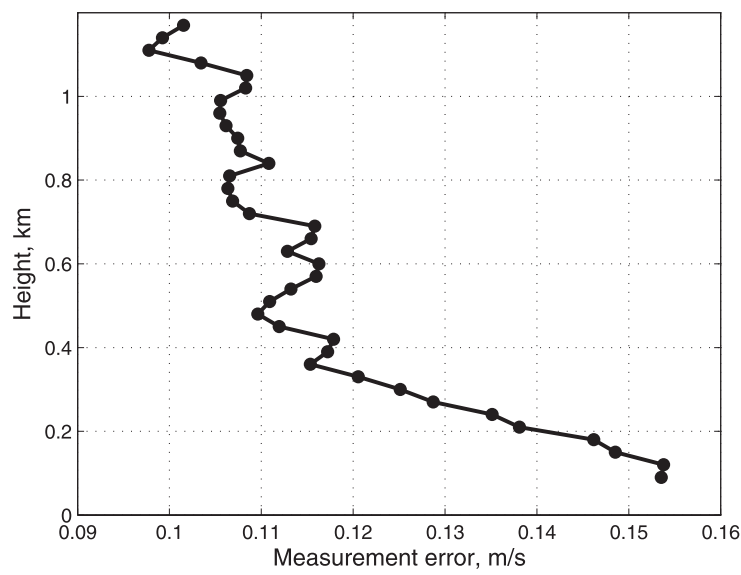

FIG. 6. (a) The first lags of the structure function of the Doppler velocity measured by the TARA radar. (b) The profile of the Doppler velocity RMS error.

this dependence by a sixth-order polynomial with coefficients changing with the height. Another imposed restriction, $\varphi(\langle Z\rangle)<0$, is used as well. An example of the approximation is shown in Figs. $4 a$ and $4 c$. One can see that the approximation is of a high degree of quality. This approach makes it possible to filter out the averaged effects of the droplet sedimentation from the vertical Doppler velocity observations data and to obtain the residual velocity field $U(h, t)=V(h, t)-\varphi(h,\langle Z\rangle)$. The mean value of this field is equal to zero, but its variance still depends on the reflectivity, as well as on the errors in the velocity measurements.

\section{b. Separation within the residual velocity field}

Figures $7 \mathrm{a}$ and $7 \mathrm{~b}$ shows an example of the residual velocity field at two height levels calculated using the observed data. Although the correlation between the residual velocity and the reflectivity is very small, the residual (a)

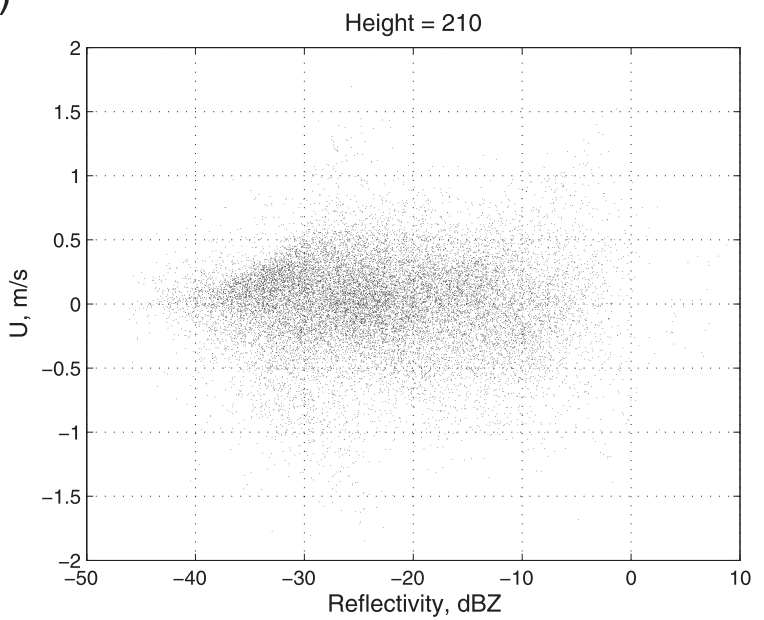

(b)

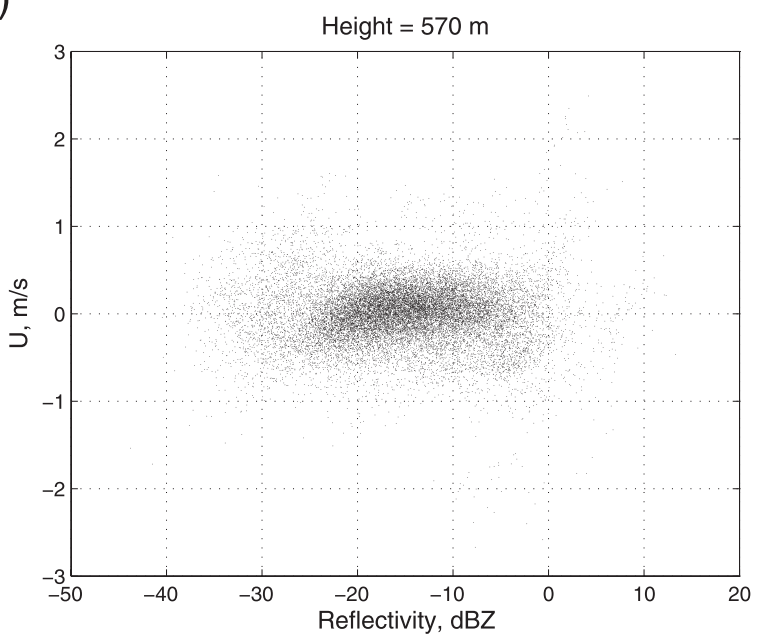

FIG. 7. Scattering diagram of the residual velocity vs the radar reflectivity at (a) 210 and (b) $570 \mathrm{~m}$.

velocity field is still affected by the droplet sedimentation velocity, due to sedimentation velocity fluctuations related to the spatial variations of the DSDs in the clouds. Namely, the RMS variance of the field depends on the reflectivity (such a dependence can also be seen in Fig. 4).

We will separate the residual velocity field $U(h, t)=$ $W(h, t)+V_{g}^{\prime}(h, t)$ into the fields of the air velocity fluctuations, $W(h, t)$, and the sedimentation velocity fluctuations, $V_{g}^{\prime}(h, t)=V_{g}(h, t)-\left\langle V_{g}(h,\langle Z\rangle)\right\rangle$, and, at the same time, we will compensate the average dependence of the variation on the reflectivity, $\sqrt{\left\langle U^{2}\right\rangle}=\theta(Z)$, as shown in Fig. 4. (This dependence is approximated using a sixthorder polynomial and is shown in Fig. 4 by the dashed lines.)

Let us define the linear estimations of the air (with respect to the measured Doppler velocity) and sedimentation velocity fluctuations for every height level $h$ that obey the condition $\hat{W}(h, t)+\hat{V}_{g}^{\prime}(h, t)=U(h, t)$ : 


$$
\begin{aligned}
& \hat{W}(h, t)=a[Z(h, t)] U(h, t) \quad \text { and } \\
& \hat{V}_{g}^{\prime}(h, t)=\{1-a[Z(h, t)]\} U(h, t),
\end{aligned}
$$

where the weight factors $a[Z(h, t)]$ are the function of the radar reflectivity. Estimations (8a) and (8b) actually represent the required retrievals. The average values of the estimations along the horizontal direction are equal to zero:

$$
\frac{1}{N} \sum_{k=1}^{N} \hat{W}\left(h, t_{k}\right)=\frac{1}{N} \sum_{k=1}^{N}\left(h, t_{k}\right)=0
$$

The weight factors $a[Z(h, t)]$ are chosen so that, on the one hand, they can compensate the mean dependence of $U(h, t)$ variance on the reflectivity, and on the other hand, so that they provide the given correlation coefficient $\rho$ between $\hat{W}(h, t)$ and $\hat{V}_{g}^{\prime}(h, t)$. We choose the factors $a[Z(h, t)]$ that are proportional to the inverse mean dependence $\theta(Z)$, so that $a(Z)=a_{0} / \theta(Z)$, where $a_{0}$ is a constant. The variance of the estimations $\hat{W}(h, t)$ and $\hat{V}_{g}^{\prime}(h, t)$, the covariance between them, and the correlation coefficient are derived in appendix B. If we take into account a nonzero correlation coefficient between the velocity components, $\rho \neq 0$, the equation for the constant $a_{0}$ has the form (see appendix B)

$$
a_{0}=\frac{\sum_{k=1}^{N} \theta_{k}^{-1}}{\sum_{k=1}^{N} \theta_{k}^{-2}}-\frac{\rho}{\frac{1}{N} \sum_{k=1}^{N} \theta_{k}^{-2}} \sqrt{\frac{\frac{1}{N} \sum_{k=1}^{N} \theta_{k}^{-2}-\left(\frac{1}{N} \sum_{k=1}^{N} \theta_{k}^{-1}\right)^{2}}{1-\rho^{2}}} .
$$

We do not know a priori the real correlation coefficient $\rho$, but we believe that its typical value is small. (The model demonstrates the maximum values to be about 0.2: see Fig. 5.) In addition, further calculations show that different small values of $\rho$ do not change the results significantly. Therefore, we use the weight factors corresponding to a zero correlation coefficient:

$$
a_{0}=\frac{\sum_{k=1}^{N} \theta^{-1}\left[Z\left(h, t_{k}\right)\right]}{\sum_{k=1}^{N} \theta^{-2}\left[Z\left(h, t_{k}\right)\right]} .
$$

Note that utilization of (10b) leads to the following formula:

$$
\left\langle\hat{W}^{2}(h)\right\rangle+\left\langle\hat{V}_{g}^{\prime 2}(h)\right\rangle=\left\langle U^{2}(h)\right\rangle .
$$

In most (but not all) cases, the factors reach their maximum at low reflectivities, which indicates the tendency of the sedimentation velocity to zero with the decrease in cloud droplet size. Hence, in the case of small reflectivity, the weights $a[Z(h, t)]$ are higher. It is interesting to note that in the case where the variance does not change with $Z$ in the residual field, $\theta(Z)=$ const and all the weight factors are equal to unity; that is, the residual field fully represents the air velocity fluctuations.

\section{Verification of the method using the model of the cloud-topped BL}

The space-time fields of the retrievals of the air velocity, $\hat{W}(h, t)$, and of the drop sedimentation velocity, $\hat{V}_{g}(h, t)=\bar{V}_{g}[Z(h, t)]+\hat{V}_{g}^{\prime}(h, t)$, obtained on the basis of the Cabauw data are shown in Fig. 8. One can see several features of the fields that are consistent with the nature of the clouds. For instance, the sedimentation velocity reaches its maximum in the high radar reflectivity zone where large drops are concentrated. The mean values of the sedimentation velocity $\left(2 \mathrm{~m} \mathrm{~s}^{-1}\right)$ correspond to a drop radius of $200 \mu \mathrm{m}$, which is the typical size of drizzle drops (e.g., Pinsky et al. 2008). The maximum sedimentation velocity reaches $4 \mathrm{~m} \mathrm{~s}^{-1}$, which corresponds to drizzle drops of $500-\mu \mathrm{m}$ radius. In zones with low radar reflectivity, the values of the sedimentation velocities are negligibly small. Similarly, the retrieved field of the air velocity indicates very low values at the lower and upper boundaries. The fluctuations in the air velocity range from -1.5 to $1.5 \mathrm{~m} \mathrm{~s}^{-1}$, which is a typical range for stratocumulus clouds (e.g., Siebert et al. 2006). One can see that the retrieved air velocity fluctuations are symmetrical with respect to zero.

Figure 9 (top) shows the scattering diagrams: the retrieved vertical air velocities versus the true vertical velocities (i.e., calculated by the model) at two height levels: 210 and $570 \mathrm{~m}$. In our opinion, the results can be considered to be quite encouraging, since the scattering is not large $\left(\sim 0.3 \mathrm{~m} \mathrm{~s}^{-1}\right)$ and no systematic deviation is found. The coefficient of the correlation is above 0.96 at both levels. This scattering seems to be related mainly to the DSD variance. Taking into account that the information used was limited (only the zero and first moments of the Doppler spectrum), one can hardly expect to obtain a lower scattering.

We compared the method proposed in the study with the more advanced method developed by Shupe et al. (2008) using information from the Doppler spectrum. The algorithm of Shupe et al. (2008) was implemented into the model and the vertical air velocity retrieved by this 
Reflectivity, dBZ

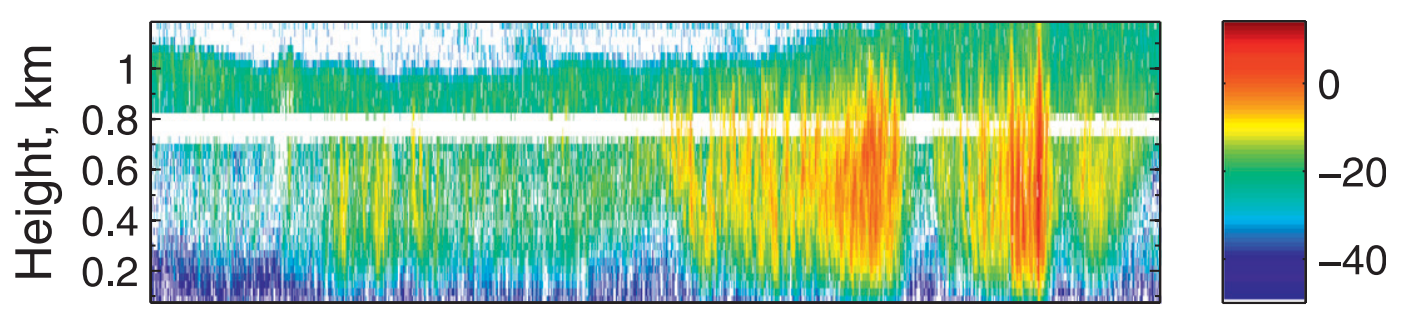

\section{Sedimentation velocity, $\mathrm{m} / \mathrm{s}$}

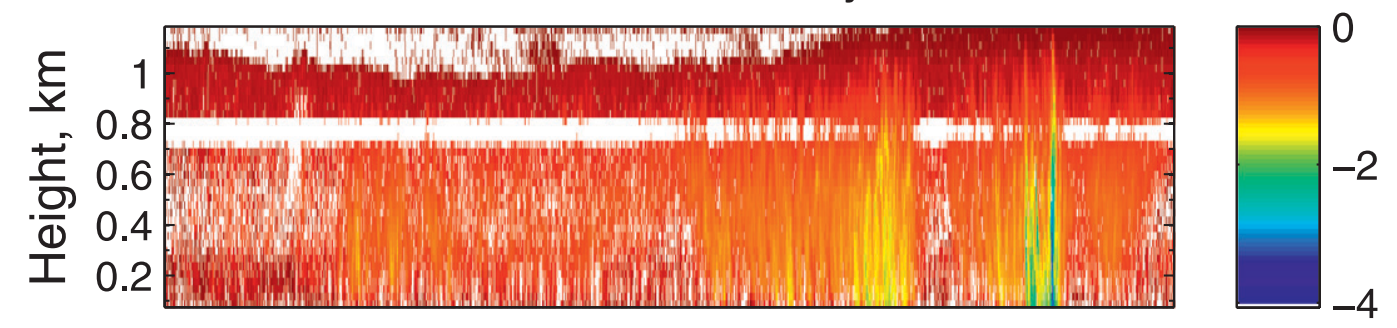

\section{Air velocity, $\mathrm{m} / \mathrm{s}$}

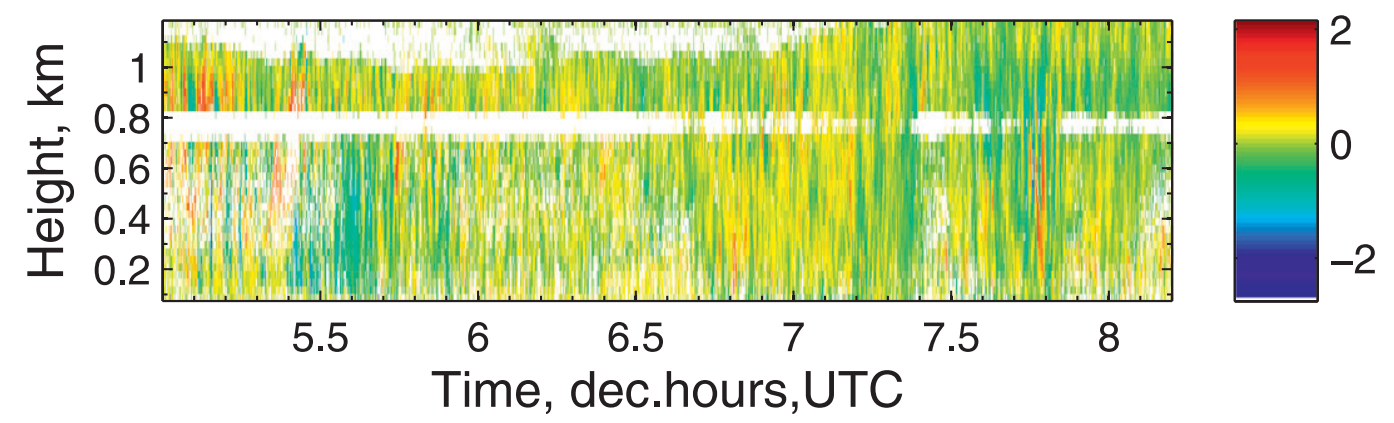

FIG. 8. Fields of the (top) radar reflectivity, (middle) retrieved sedimentation velocity, and (bottom) retrieved air velocity obtained from the Cabauw observation data.

method was compared both with the model air velocity and with the velocity retrieved by the method proposed in the present paper (Fig. 9, middle and bottom). One can see that the air velocity retrieved using Shupe et al.'s (2008) method agrees with the model velocity better that that proposed in the present study. Shupe et al.'s (2008) method shows a coefficient of correlation that exceeds 0.98 at both the 210 - and 570-m height levels. Note that the scattering in the diagrams related to the Shupe et al. (2008) method increases with decreasing height. In addition, the scattering diagrams at $210 \mathrm{~m}$ and below reveal systematic deviations: Shupe et al.'s (2008) method underestimates the air velocity. We attribute this result to a specific feature of the approach, according to which the reference point in the Doppler spectrum corresponds to the smallest droplets whose velocity is assumed equal to the air velocity. At low levels, DSDs may not contain small droplets whose velocities could be equal to that of the air. The bottom-most panels in Fig. 9 show quite good agreement between the air velocities retrieved by the two methods. Note that the method developed by Shupe et al. (2008) uses the shape of the Doppler spectrum as the input. Such information is often not available, as, for instance, was the case in the Cabauw measurements. A reasonably good level of agreement between the retrieved air velocities and those generated by the model, as well as good agreement among the vertical air velocities retrieved by the methods, make it reasonable to use the method proposed in the present study, at least in cases when only the first moment of the Doppler spectrum is available.

Figure 10 shows the vertical profiles of the RMS vertical air velocity fluctuations, $\sqrt{\left\langle\hat{W}^{2}(h)\right\rangle}$, calculated directly by the model and those obtained using the retrieval method. The retrieved vertical profiles of the skewness and kurtosis are presented in the right panel of Fig. 10. One can see that the profile of the retrieved RMS vertical air velocity is quite close to that in the model. The 

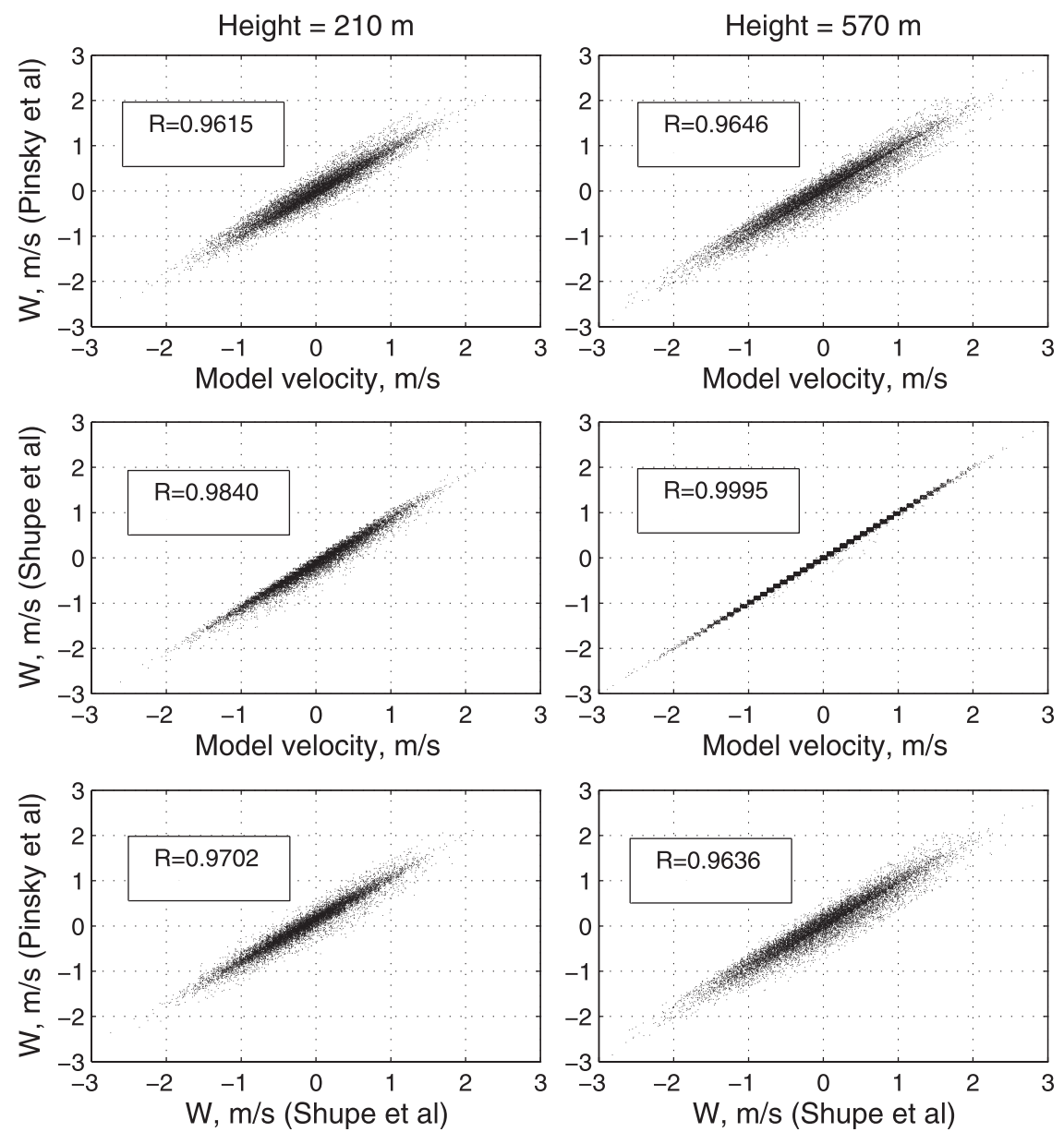

FIG. 9. Scattering diagrams at (left) the 210- and (right) 570-m levels: (top) retrieved vertical air velocities according to the proposed method vs the model's vertical air velocity, (middle) retrieved vertical air velocities using the method suggested by Shupe et al. (2008) vs the model's vertical air velocity, and (bottom) the scattering diagrams showing the comparison of these two methods.

RMS velocity $\sqrt{\left\langle\hat{W}^{2}(h)\right\rangle}$ tends to zero at the surface and near cloud top, which indicates that the method enables us to confidently eliminate the contribution of the drop sedimentation velocity (seen in Fig. 3) to the Doppler velocity. The statistical errors are expressed mainly by the bias, which is maximal in the middle of the BL, where it does not exceed $8 \%$. In spite of the fact that the RMS error of the velocity estimation in every scattering volume is as large as $20-30 \mathrm{~cm} \mathrm{~s}^{-1}$ (Fig. 9), the horizontally averaged values [profiles of $\sqrt{\left\langle\hat{W}^{2}(h)\right\rangle}$, higher moments] are obtained with quite small errors (see the bars in the left panel of Fig. 10). The uncertainty ranges of the RMS $( \pm \sigma)$ in Fig. 10 were obtained by the integration of the square of the correlation function of $\hat{W} ; \sigma$ does not exceed $6 \mathrm{~cm} \mathrm{~s}^{-1}$. Note that the model data does not contain measurement noise. The errors in such profiles obtained by applying the method to the real Doppler data are larger.
The skewness and the kurtosis of the retrieved velocity fields are equal to 0 and 3, respectively; this corresponds to the normal distribution of the air velocity, which was assumed for the development of the turbulentlike velocity field in the model.

Figures 9 and 10 show that the proposed algorithm allows us to retrieve the velocity field and, correspondingly, makes it possible to investigate the turbulent structure of the cloud-capped mixed layer using a vertically pointed Doppler S-band radar. The method also provides some information about the mean sedimentation velocity, which, in combination with the radar reflectivity, can be used for the analysis of the microphysical cloud structure. In this study, we present only part of the results concerning the BL turbulent structure.

To test the sensitivity of our results to the averaging procedures, supplemental calculations have been carried out. In the tests the order of approximating the 

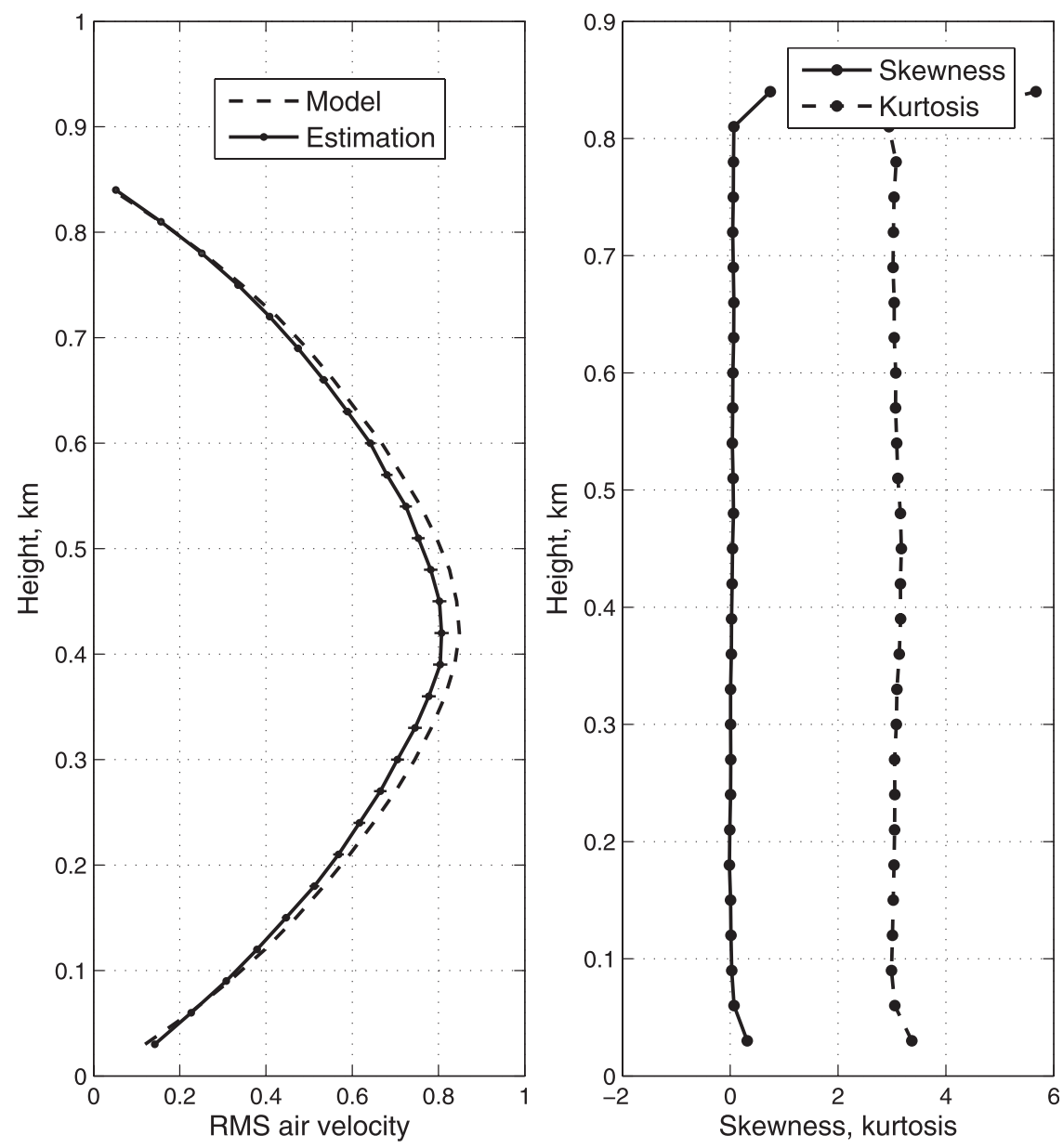

FIG. 10. (left) Vertical profiles of the RMS vertical air velocity fluctuations calculated by the model and those retrieved. (right) Skewness and kurtosis of the retrieved profiles.

polynomials was decreased/increased by 1 . We also tested the sensitivity of results to the choice of the dependence of the weighting coefficients on the reflectivity [instead of $a \sim 1 / \theta(Z)$, the dependence $a \sim 1 / \theta^{2}(Z)$ was used]. For the model fields, the sensitivity to these changes was negligible. The results obtained using the real measurements demonstrated a difference of $2-4 \mathrm{~cm} \mathrm{~s}^{-1}$ for the evaluation of the vertical velocity.

\section{BL turbulent structure}

\section{a. Statistical characteristic of the air velocity field}

Profiles of the statistical moments of the air velocity field, namely, the RMS vertical velocity $\left\langle W^{2}(h)\right\rangle^{1 / 2}$, the skewness $S=\left\langle W^{3}(h)\right\rangle /\left\langle W^{2}(h)\right\rangle^{3 / 2}$, and the kurtosis $F=$ $\left\langle W^{4}(h)\right\rangle /\left\langle W^{2}(h)\right\rangle^{2}$, are shown in Fig. 11. The calculation of these quantities includes an additional correction of the measurement white errors shown in Fig. 6. The vertical velocity fluctuations tend to zero toward the surface and just above cloud top. The latter can be attributed to the presence of a very stable inversion layer, which damps small-scale vertical turbulent velocity fluctuations. The maximum value of the RMS velocity fluctuations is not pronounced and reaches about $40 \mathrm{~cm} \mathrm{~s}^{-1}$, which can be considered to be a typical value for a developing (early morning) boundary layer. The profile of the RMS air velocity resembles that presented by Lothon et al. (2005; Fig. 7) and characterized by a fast increase in the lower 100-200-m layer, almost constant values up to $\sim 800-\mathrm{m}$ level, and a fast decrease close to the inversion layer.

The profile of the skewness qualitatively agrees with that presented by Stevens et al. (2005a): the skewness is positive near the surface and becomes slightly negative near the cloud base. (The positive deviation at $800 \mathrm{~m}$ is caused by a technical measurement problem.) In general, the skewness is close to zero, which means the velocity fluctuation distribution is close to being symmetric. 

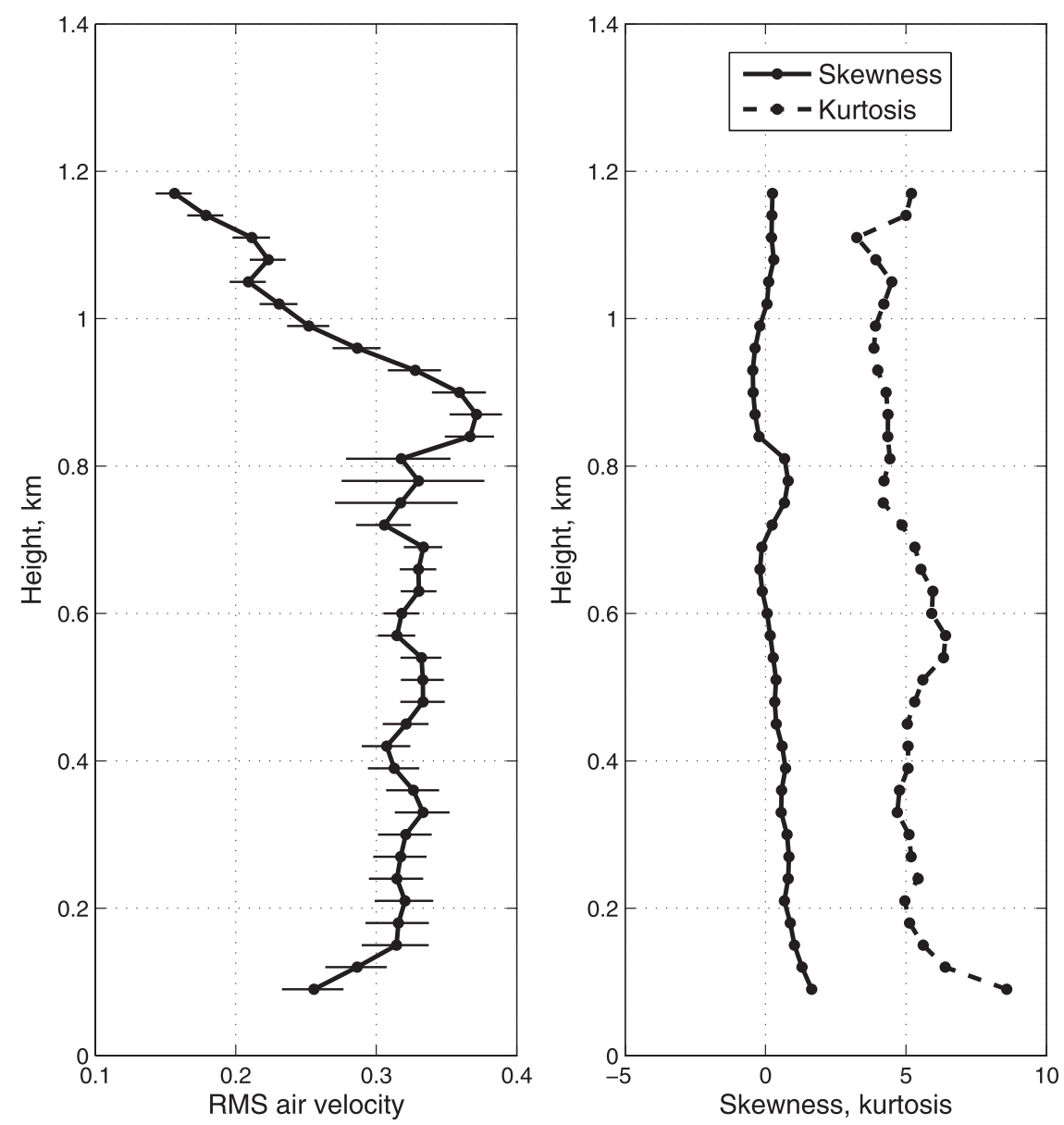

FIG. 11. (left) Height dependence of the vertical air velocity RMS with error bars and (right) skewness and kurtosis of the profiles obtained from the Cabauw observation data.

The kurtosis has values of about $4-5$, indicating that the distribution of the air velocity field is quite close to the normal distribution. In the vicinity of the surface (in the lowest layer of 150-m depth), the velocity distribution differs from the normal one. It is possible that the large kurtosis found below $100 \mathrm{~m}$ is related to errors in the radar measurements at these low levels.

Figure 12 shows the normalized spatial correlation functions of the air velocity field at different heights. The Taylor hypothesis of frozen turbulence has been used for their calculations. The conversion of the time variations of the radar data to the horizontal spatial domain has been done using the mean horizontal wind velocity profile, which was interpolated from the radiosonde data nearest in time. According to these data, the mean horizontal velocity increases over height practically linearly from $6.5 \mathrm{~m} \mathrm{~s}^{-1}$ at the surface up to $10 \mathrm{~m} \mathrm{~s}^{-1}$ at $1000-\mathrm{m}$ height.

Near the surface and near the inversion level (cloud top), the radius of correlation is about $200 \mathrm{~m}$. In the middle of the boundary layer, the correlation function shows an oscillation with the $600-\mathrm{m}$ period. Such periodicity can be attributed to the presence of large eddies, which is a typical feature of the velocity field structure in the BL (Bizova et al. 1989; LeMone 1973; Ivanov and Khain 1975; Stevens et al. 2005a,b). The results obtained in our study agree well with measurements taken on a 300m-high meteorological tower in Obninsk, Russia (Bizova et al. 1989), according to which the existence of large eddies (or convective cells) becomes pronounced in the velocity spectrum and the temperature spectrum above 150-200 m. Below this height, the spectra typical of small-scale turbulence dominate.

\section{b. Turbulent parameters}

The turbulent structure of the velocity field is usually described using the structure functions. Usually, the dependence of a structure function on the distance $r$ is represented by a power law. In homogeneous and isotropic 


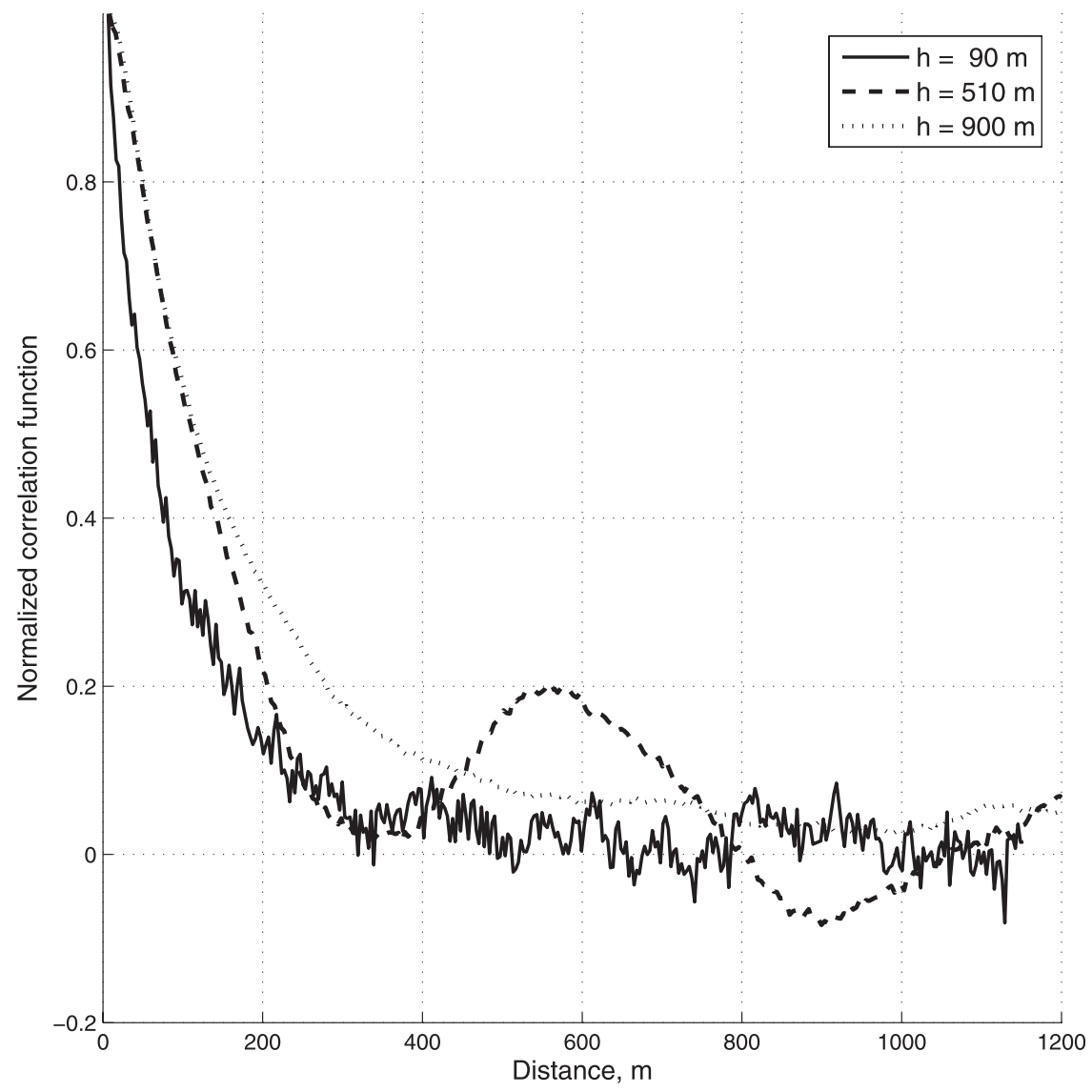

FIG. 12. Normalized correlation function of the air velocity field retrieved from the Cabauw observations, calculated at different heights in the cloud-capped BL.

turbulence, the lateral structure function within an inertial subrange has the form (Monin and Yaglom 1975)

$$
D_{\mathrm{NN}}(r)=\frac{4}{3} C(\varepsilon r)^{2 / 3}
$$

where $\varepsilon$ is the turbulent dissipation rate and $C=2$ is Kolmogorov's constant. However, in the vicinity of the surface one can expect deviation from the Kolmogorov law of isotropic turbulence. One can also expect the the dependence of turbulent properties on height, which should tend to the Kolmogorov law at higher distances from the surface. It means that near the surface we have an additional spatial scale, namely, the distance from the surface. Accordingly, we assume that the structure function in the atmospheric $\mathrm{BL}$ can be represented as the power function with a height-dependent exponent, so

$$
D_{\mathrm{NN}}(r)=A(h) r^{\beta(h)},
$$

where $\beta(h)$ is the scaling parameter that tends to $2 / 3$ with a height increase within the surface layer. Equation (13) was used by Bizova et al. (1989). Figure 13 (left) presents an example of the structure functions at three height levels. Figure 13 indicates the presence of three zones in the height-dependent structure functions. At small distances $r$, the radar smoothing decreases the slope. Within the inertial turbulence range (20-120 m), one can see the clear linear slope of the functions in the $\log -\log$ coordinates. At the distances of several hundred meters or more, the structure function tends to plateau, which corresponds to the correlation function's tendency to zero. The oscillations of the structure function, in the middle part of the boundary layer, seen in the plateau, can be attributed to the existence of the large eddies, as was mentioned above. One can see that the slope of the structure functions within the inertial range depends of height. This dependence is more pronounced in Fig. 13 (right).

To evaluate the scaling, we approximated the structure functions by employing a power function within the distance range of 30-120 m, using the least squares method. Below $30 \mathrm{~m}$, the spatial smoothing inside the radar reflection volume affects the results. At distances 

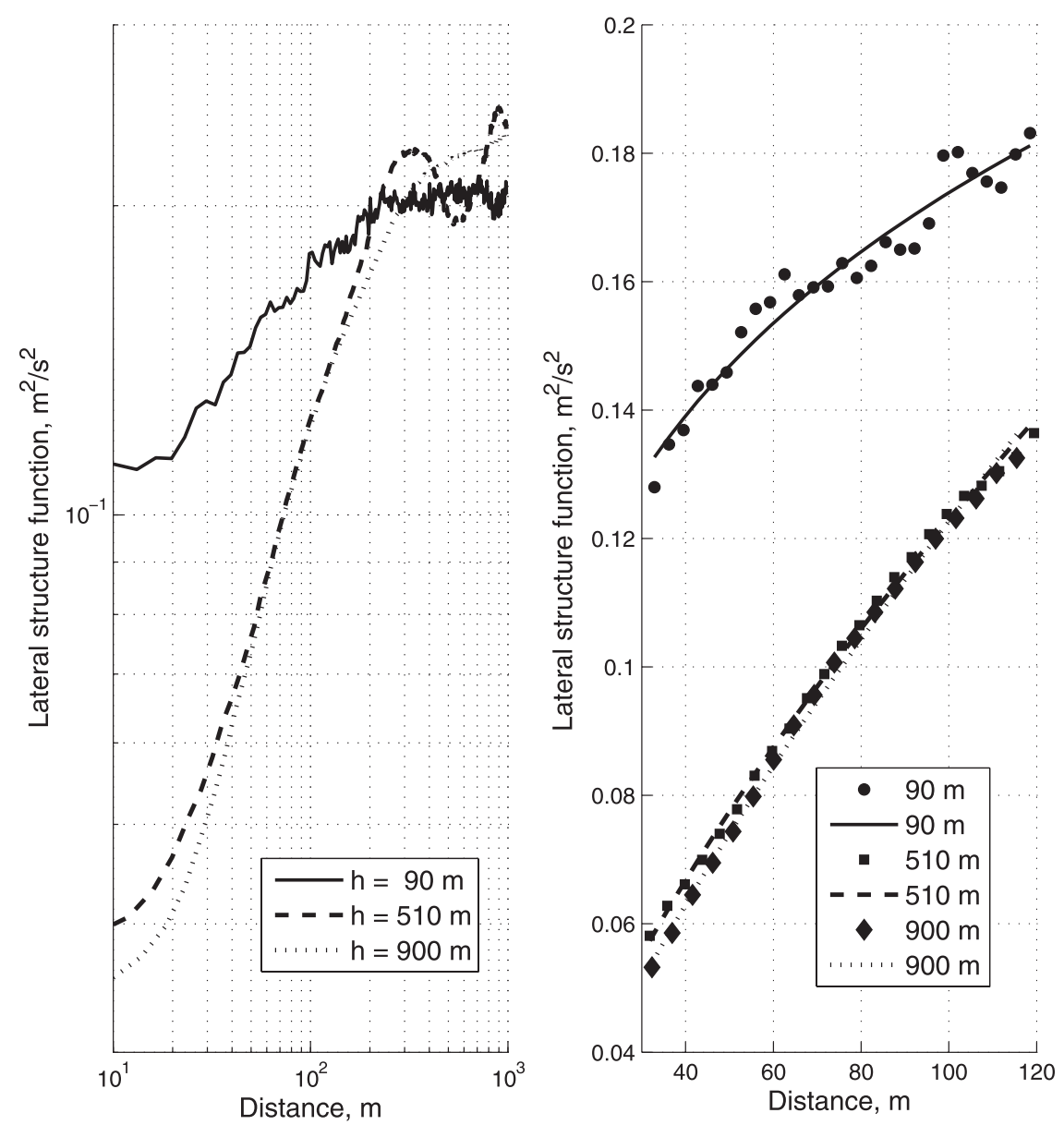

FIG. 13. The structure functions at different height levels in (left) log-log and (right) linear coordinates. The lines in the right panel show the approximation of the structure functions by the power function in (13).

larger than $120 \mathrm{~m}$, the results are affected by large (nonturbulent) eddies. The profile of scaling $\beta(h)$ is shown in the middle panel of Fig. 14.

Using this approximation, the integral scale of turbulence was estimated as

$$
\begin{aligned}
L_{0}(h) & =\frac{1}{\left\langle W^{2}(h)\right\rangle} \int_{0}^{r_{0}(h)} B(h, r) d r \\
& =\frac{1}{\left\langle W^{2}(h)\right\rangle} \int_{0}^{r_{0}(h)}\left[\left\langle W^{2}(h)\right\rangle-\frac{1}{2} D_{\mathrm{NN}}(h, r)\right] d r \\
& =r_{0}(h)-\frac{1}{2\left\langle W^{2}(h)\right\rangle} \int_{0}^{r_{0}(h)} A(h) r^{\beta(h)} d r,
\end{aligned}
$$

where $B(r)$ is the lateral correlation function determined by turbulence and $r_{0}(h)=\left[2\left\langle W^{2}(h)\right\rangle / A(h)\right]^{1 / \beta(h)}$ is the distance at which the correlation function is equal to zero. From (13) one can obtain

$$
L_{0}(h)=\frac{\beta(h)}{1+\beta(h)}\left[\frac{2\left\langle W^{2}(h)\right\rangle}{A(h)}\right]^{1 / \beta(h)} .
$$

The profile of the integral scale $L_{0}(h)$ is shown in the left panel in Fig. 14. The integral turbulence scale was found to be of the order of $100 \mathrm{~m}$, which is the typical value for atmospheric turbulence. The nearly linear decrease of the scale toward the surface within the lowest 200-m layer can be attributed to the decrease in the distance from the surface. In the surface layer the distance above the surface is the natural spatial scale determining the maximum sizes of turbulent vortices. This pattern of behavior agrees well with the theory of the surface layer, according to which the mixing length is the linear function of the height (e.g., Garratt 1992). The middle panel in Fig. 14 shows that the scaling exponent $2 / 3$ is observed at heights above $400-500 \mathrm{~m}$. This result indicates that turbulence in the BL can be considered isotropic (obeying 

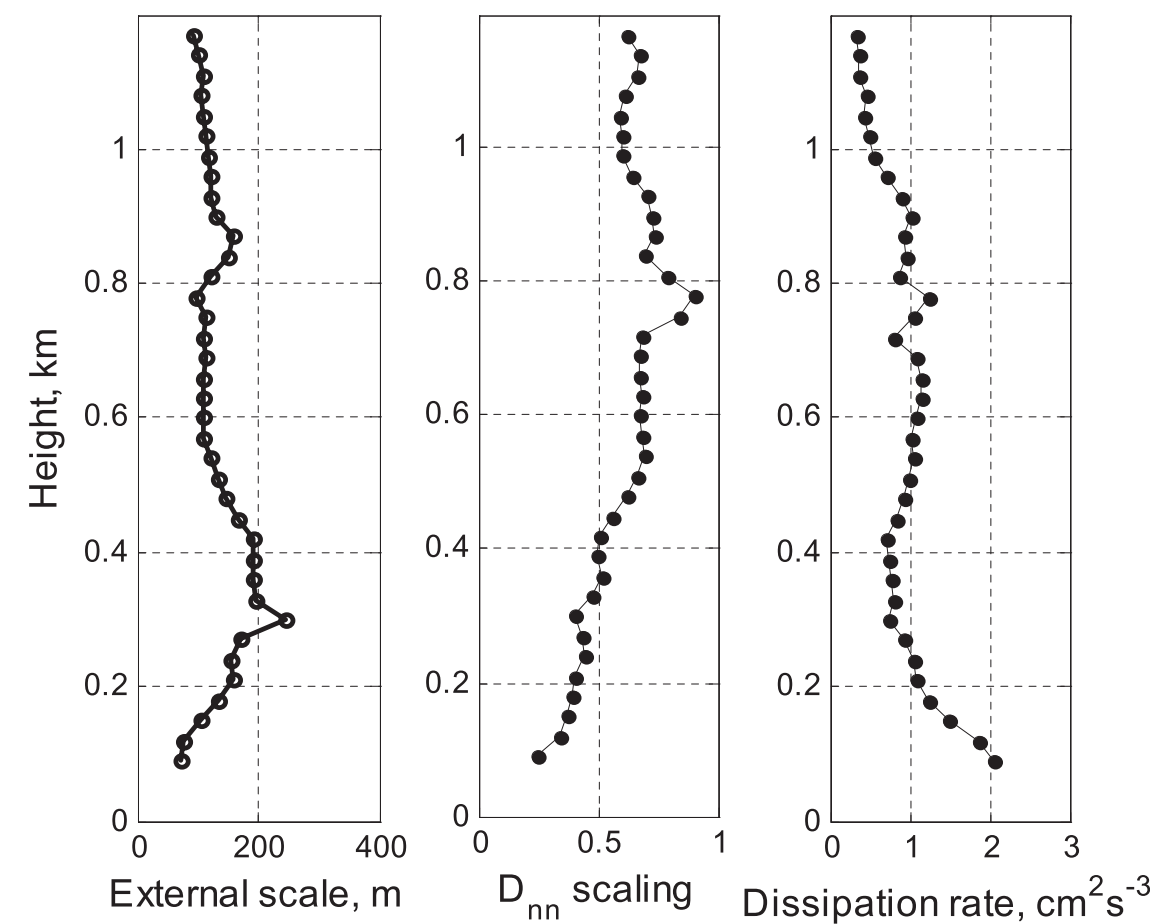

FIG. 14. Vertical profiles of the (left) turbulent integral scale, (middle) structure function scaling, and (right) turbulent dissipation rate.

the Kolmogorov 2/3 law) only within the upper part of the BL. Below the 400-m level, the slope of the structure function decreases down to 0.2 , which corresponds to the -1.2 slope of the spectrum. We attribute the decrease in the $D_{\mathrm{NN}}(r)$ slope to the nonisotropy of the turbulence in the lower part of the BL below $400 \mathrm{~m}$. The rate of the anisotropy increases with the decrease in height above the surface. This assumption seems to be reasonable from a physical point of view. The turbulent kinetic energy (TKE) can be presented as the sum of the three components related to the mean squares of the corresponding air velocity component fluctuations. It is known that in the TKE equation pressure fluctuations are responsible for the distribution of turbulent energy between these components (Garratt 1992). Near the surface, fluctuations of vertical velocity are much smaller than those of the horizontal velocity; that is, the pressure force generates mainly horizontal velocity fluctuations. In this layer, the component of the TKE related to the vertical velocity fluctuations is small because the work of the pressure $\left\langle p^{\prime} w^{\prime}\right\rangle$ in the vertical direction is negligible. With an increase in the distance above the surface, the generation of vertical velocity fluctuations increases and the pressure force redistributes the TKE among the vertical and horizontal directions making the turbulence isotropic at heights above $400 \mathrm{~m}$. The anisotropy of the turbulence near the surface reflects the change in the rate of the energy flux from large to smaller scales. This suggests the existence of a close relationship between the anisotropy of the turbulence and the structure function scaling.

Hence, we assume that the structure function $D_{\mathrm{NN}}(r)$ can be approximated as

$$
D_{\mathrm{NN}}(r)=\frac{4}{3} C(\varepsilon r)^{2 / 3}\left(\frac{r}{L_{0}}\right)^{\gamma},
$$

where $\gamma=\beta-2 / 3$. If $\gamma=0$, one obtains the formula for the structure function for homogeneous and isotropic turbulence (12). The estimation of the turbulent dissipation rate profile $\varepsilon$ can be derived from Eqs. (13) and (16):

$$
\varepsilon(h)=\left[\frac{3}{4} \frac{A(h)}{C}\right]^{3 / 2}\left(L_{0}\right)^{3 / 2 \beta(h)-1}
$$

Equations (16) and (17) obey the similarity theory. Application of these formulas gives a very good approximation of the results, as shown in Fig. 13. The right panel in Fig. 14 shows the vertical profile of the dissipation rate in the $\mathrm{BL}$, which was derived from the radar observation at Cabauw. The retrieved values in this case are quite small, on the order of $1-2 \mathrm{~cm}^{2} \mathrm{~s}^{-3}$. Some growth in the dissipation rate toward the surface is seen in Fig. 14 
and can be attributed to the increase in the wind shear near the surface. The values of the dissipation rate in the cloud-capped mixed layer usually vary from 0.5 to $50 \mathrm{~cm}^{2} \mathrm{~s}^{-3}$ (e.g., Siebert et al. 2006; Muschinski et al. 2004). The comparatively low dissipation rate in the Cabauw case considered here can be attributed to the fact that BL development was analyzed during morning hours (0500-0800 LT), when turbulence was weak. Drizzle started forming only after 0645 LT, indicating that the developed mixed layer formed relatively late. In DYCOMPS-II, the maritime BL remained developed during the entire period of observation. This fact may explain the higher dissipation rate values of $5 \mathrm{~cm}^{2} \mathrm{~s}^{-3}$ reported by Lothon et al. (2005).

Note that Siebert et al. (2006) showed that the smallness of the mean values of the dissipation rate does not exclude the probability of the high values of the dissipation rate in particular zones of the BL.

\section{Conclusions}

A method for retrieving air velocity fluctuations in the cloud-capped mixed layer using radar reflectivity and the Doppler velocity is proposed. The efficiency of the method was demonstrated using the data obtained by the TARA radar located in Cabauw at 0500 0812 UTC 8 May 2004, and was tested by means of a detailed trajectory ensemble model of the cloudcapped BL. It has been shown that the method is able to accurately retrieve the air turbulent velocity field from the vertically pointed Doppler radar observations and to filter out the influence of the falling cloud droplets and drizzle, at least in cases of nondrizzling and weakly drizzling stratocumulus clouds. This opened the possibility of investigating in similar cases the turbulent structure of the BL, using the data obtained with a vertically pointed Doppler S-band radar. The radar reflectivity and the Doppler velocity data obtained by the TARA radar during the development of a drizzling stratocumulus cloud were used to calculate of the main characteristics of the turbulent velocity field in the BL.

Analysis of the correlation functions indicates the existence of large eddies in the BL, with the characteristic horizontal scale of about $600 \mathrm{~m}$. Our analysis of the scaling of the structure functions at different heights indicates that turbulence above $400 \mathrm{~m}$ can be considered isotropic (the slope parameter of the structure function is close to $2 / 3$ ). Below the $400-\mathrm{m} \mathrm{level,} \mathrm{the} \mathrm{scaling} \mathrm{de-}$ creases down to 0.2 . We suppose that this decrease reflects the fact that turbulence becomes anisotropic below this height. The rate of anisotropy increases with a decrease in the distance above the surface. The relationship between the rate of turbulence anisotropy and the structure function scaling near the surface requires additional investigation.

The vertical profiles of the dissipation rate have been calculated. The results indicate that the averaged values of the dissipation rate in the mixing layer were about $1 \mathrm{~cm}^{2} \mathrm{~s}^{-3}$. This comparatively low value can be attributed to the fact that the time period chosen for the analysis was from 0500 to $0800 \mathrm{LT}$, when the mixing layer was not fully developed and the convection was not strong. This low value of the dissipation rate agrees with the comparatively low value of the evaluated vertical velocity fluctuations with maximum of $0.4-0.5 \mathrm{~m} \mathrm{~s}^{-1}$. More data should be analyzed to better verify the method and to understand changes in the turbulent structure of the $\mathrm{BL}$ during the drizzle formation process.

Note that the model of the cloud-capped BL served as an effective tool for verification of the proposed retrieval algorithm. In this connection, we see an important role that this model can play in the investigation of BL structures and cloud microphysics. We suggest that investigations of the cloud-capped BL be performed within a closed "observation-simulation-observation" cycle, as illustrated in the scheme presented in Fig. 15. As the initial observational data for the model initialization, one can use sounding data, aerosol data, and Doppler radar data (the radar reflectivity and the Doppler vertical velocity). In addition, lidar data can be used to estimate the aerosol concentration in the BL, to determine the height of cloud base, etc. The application of the retrieval method that is described in this paper allows us to gain new insight into the turbulent structure of the BL. The statistical properties of the retrieved air velocity should be assimilated into the model to generate the turbulentlike velocity field (see Pinsky et al. 2008 for detail). Assimilation of the dynamics means that parameters of the model velocity field have the same energy and correlation properties as those of the observed (retrieved) velocity field. The parameters of the aerosol size distributions and the sounding data (temperature, dewpoint, and wind velocity profiles) can also be easily assimilated by the model. As a result, the model can be tuned to observational input. The results of the simulations should be compared with the corresponding simultaneously measured microphysical, radar, and lidar cloud characteristics. Thus, the cloud-capped BL model can serve as an efficient connecting link between different types of observed data (radar, lidar, aircraft, etc.). Results of the comparison, on the one hand, will contribute to a better understanding of cloud microphysical processes and, on the other hand, will make it possible to improve both the interpretation of remote sensing data and the retrieval algorithms. 


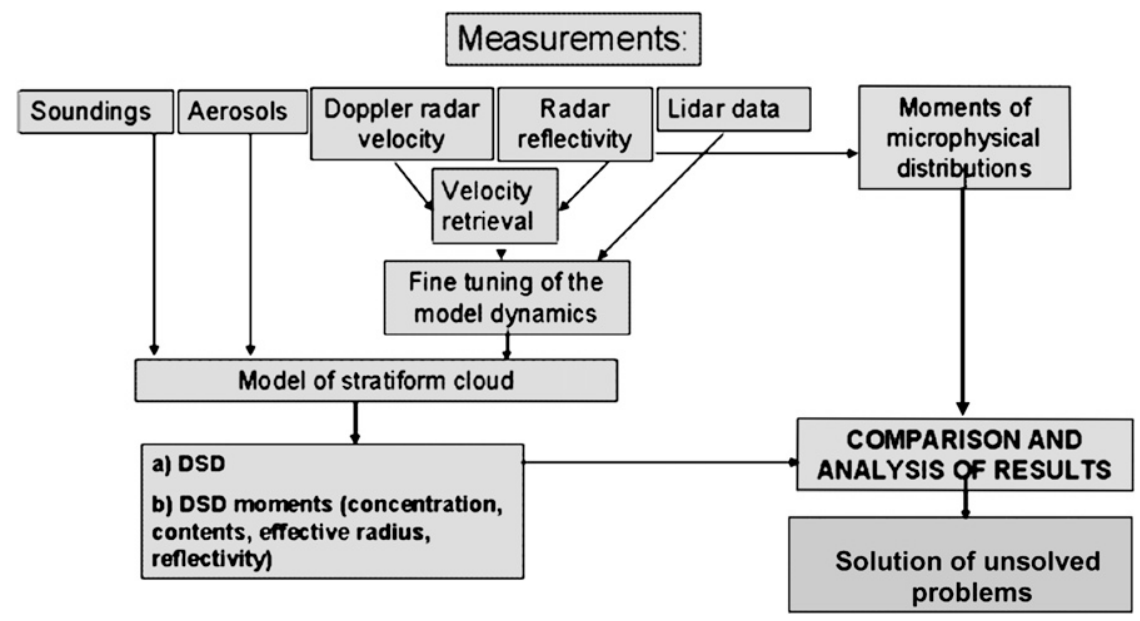

FIG. 15. Suggested coupling of the model and observations aimed at improving the retrieval algorithms and investigating the cloud microphysics.

Acknowledgments. The study was carried out under the support of the Israel Sciences Foundation (Grant 950/07) and of the Netherlands Space Agency (SRON) and the Dutch National Research Program Climate Changes Spatial Planning.

\section{APPENDIX A}

\section{A Brief Description of the Trajectory Ensemble Cloud Model}

The model is described in detail by Pinsky et al. (2008). In the model, the boundary layer (BL) is fully covered by a great number (in our case 6500) of Lagrangian air parcels, each with a characteristic size of about $40 \mathrm{~m}$ that, while moving, can contain either wet aerosols or aerosols and droplets. The Lagrangian parcels are advected by the velocity field generated by the turbulent-like flow model obeying the turbulent correlation laws. The velocity field in this model is represented as a sum of a large number of harmonics of different wavelengths. The amplitude of the harmonics randomly changes over time according to the turbulent laws. The dynamic part of the model contains a set of parameters that allow for the adjustment of the statistical properties of the model airflow to observed characteristics. The main dynamic (turbulent) parameters used for the adjustment are the vertical profile of the RMS turbulent velocity and the correlation (or structure) function of the vertical velocity in the horizontal direction.

Calculation of microphysical processes in each Lagrangian parcel includes the calculation of the growth-decrease of droplets and aerosols by condensation-evaporation performed with high accuracy. The mass of the aerosols inside droplets is also calculated. When droplets evaporate, aerosol particles return into the air. The accurate description of drop collisions is possible, since the stochastic collision equation for DSD is solved on a highresolution mass grid containing 500 mass bins. Droplet sedimentation is taken into account, which enables us to simulate the precipitation formation. The droplet sedimentation velocity is accurately calculated by taking into account fine factors affecting the sedimentation velocity of droplets of different sizes (Beard 1976).

The output of the numerical model includes droplet and aerosol size distributions and their moments, such as droplet concentration, droplet spectrum width, cloud water content, drizzle content, radar reflectivity, etc., calculated for each parcel. Horizontally averaged values are calculated as well.

The ability of the model to reproduce the main microphysical parameters, including the fields of drop concentration, liquid water content (LWC), DSD width, dispersion, drizzle size, and so on, was tested in detail by Magaritz et al. (2009). DSDs calculated by the model vary in time and space and significantly change at distances of a few tens of meters, which agrees with observations (e.g., Korolev 1995). The ability of the model to reproduce the $Z$-LWC diagrams observed in different field experiments is shown by Khain et al. (2008) and Pinsky et al. (2008). These properties of the model allow its application for the purposes of remote sensing and the analysis of the dynamic and microphysical properties of the BL.

In comparison with the model version described by Pinsky et al. (2008), the present version is expanded to calculate the Doppler velocity according to expressions 
(1) and (2). The size of the computational area in the horizontal direction in the present version increased from 2.5 to $12.5 \mathrm{~km}$. The height of the computational area is $900 \mathrm{~m}$. The background wind velocity was chosen to be equal to $10 \mathrm{~m} \mathrm{~s}^{-1}$. The radar reflectivity and the Doppler velocity were calculated in the column with $x=$ $12.5 \mathrm{~km}$ during $4 \mathrm{~h}$. As a result, time-height series of the radar reflectivity and the Doppler velocity were calculated, reproducing the series measured by a vertically pointed Doppler radar.

\section{APPENDIX B}

\section{Statistical Properties of the Estimation and the Weight Factor}

The moments of the estimations $\hat{W}(h, t)$ and $\hat{V}_{g}^{\prime}(h, t)$ can be derived from Eqs. (8a) and (8b). Let us define $S_{1}=$ $(1 / N) \sum_{k=1}^{N} \theta^{-1}\left[Z\left(h, t_{k}\right)\right]$ and $S_{2}=(1 / N) \sum_{k=1}^{N} \theta^{-2}\left[Z\left(h, t_{k}\right)\right]$. From (8a) and (8b), one obtains the following variances,

$$
\begin{aligned}
\left\langle\hat{W}^{2}(h)\right\rangle & =\frac{1}{N} \sum_{k=1}^{N}\left\langle a^{2}\left[Z\left(h, t_{k}\right)\right] U^{2}\left(h, t_{k}\right)\right\rangle=a_{0}^{2}\left\langle U^{2}(h)\right\rangle S_{2} \\
\left\langle\hat{V}_{g}^{\prime 2}(h)\right\rangle & =\frac{1}{N} \sum_{k=1}^{N}\left\langle\left\{1-a\left[Z\left(h, t_{k}\right)\right]\right\}^{2} U^{2}\left(h, t_{k}\right)\right\rangle \\
& =\left\langle U^{2}(h)\right\rangle\left(1-2 a_{0} S_{1}+a_{0}^{2} S_{2}\right) ;
\end{aligned}
$$

the covariance between the estimations,

$$
\begin{aligned}
\left\langle\hat{W}(h) \hat{V}_{g}^{\prime}(h)\right\rangle= & \frac{1}{N} \sum_{k=1}^{N}\left\langle a\left[Z\left(h, t_{k}\right)\right]\right. \\
& \left.\times\left\{1-a\left[Z\left(h, t_{k}\right)\right]\right\} U^{2}\left(h, t_{k}\right)\right\rangle \\
= & a_{0}\left\langle U^{2}(h)\right\rangle\left(S_{1}-a_{0} S_{2}\right) ;
\end{aligned}
$$

and the square of the correlation coefficient, $\rho^{2}$,

$$
\rho^{2}(h)=\frac{\left\langle\hat{W}(h) \hat{V}_{g}^{\prime}(h)\right\rangle^{2}}{\left\langle\hat{W}^{2}(h)\right\rangle\left\langle\hat{V}_{g}^{\prime 2}(h)\right\rangle}=\frac{\left(S_{1}-a_{0} S_{2}\right)^{2}}{S_{2}\left(1-2 a_{0} S_{1}+a_{0}^{2} S_{2}\right)} .
$$

Equation (B4) leads to a quadratic equation with respect to an unknown coefficient $a_{0}$ :

$$
S_{2}^{2}\left(1-\rho^{2}\right) a_{0}^{2}-2 S_{1} S_{2}\left(1-\rho^{2}\right) a_{0}+\left(S_{1}^{2}-S_{2} \rho^{2}\right)=0 .
$$

The solution of (B5), in case the correlation coefficient and the covariance are of to the same sign, is

$$
a_{0}=\frac{S_{1}}{S_{2}}-\frac{\rho}{S_{2}} \sqrt{\frac{S_{2}-S_{1}^{2}}{1-\rho^{2}}} .
$$

In the case of zero correlation, the coefficient $\rho=0$ and the solution is

$$
a_{0}=\frac{S_{1}}{S_{2}}
$$

\section{REFERENCES}

Babb, D., and J. Verlinde, 1999: Vertical velocity statistics in continental stratocumulus clouds as measured by a $94 \mathrm{GHz}$ radar. Geophys. Res. Lett., 26, 1177-1180.

Beard, K. V., 1976: Terminal velocity and shape of cloud and precipitation drops aloft. J. Atmos. Sci., 33, 852-864.

Bizova, N. L., V. N. Ivanov, and E. K. Garger, 1989: Turbulence in the Atmospheric Boundary Layer. Gidrometizdat, 262 pp.

Campistron, B., A. B. Long, and A. W. Huggins, 1991: A method of retrieving turbulence parameters from volume processing of single-Doppler radar measurements. J. Atmos. Oceanic Technol., 8, 491-505.

Duynkerke, P. G., H. Zhang, and P. J. Jonker, 1995: Microphysics and turbulent structure of nocturnal stratocumulus as observed during ASTEX. J. Atmos. Sci., 52, 2763-2777.

Frisch, A. S., D. H. Lenschow, C. W. Fairall, W. H. Schubert, and J. S. Gibson, 1995: Doppler radar measurements of turbulence in marine stratiform cloud during ASTEX. J. Atmos. Sci., 52, 2800-2808.

Gage, K. S., C. Williams, W. Ecklund, and P. Johnston, 1999: Use of two profilers during MCTEX for unambiguous identification of Bragg scattering and Rayleigh scattering. J. Atmos. Sci., 56, 3679-3691.

Garratt, J. R., 1992: The Atmospheric Boundary Layer. Cambridge University Press, $316 \mathrm{pp}$.

Heijnen, S. H., L. P. Ligthart, and H. W. J. Russchenberg, 2000: First measurements with TARA; an S-band transportable atmospheric radar. Phys. Chem. Earth II, 25, 995-998.

Ivanov, V. N., and A. P. Khain, 1975: On dry and moist cellular convection in the atmosphere. Atmos. Oceanic Phys., 11, 1211-1219.

Khain, A., M. Pinsky, L. Magaritz, O. Krasnov, and H. W. J. Russchenberg, 2008: Combined observational and model investigation of $Z$-LWC relationship in stratocumulus clouds. J. Appl. Meteor. Climatol., 47, 591-606.

Kollias, P., and B. Albrecht, 2000: The turbulence structure in a continental stratocumulus cloud from millimeter-wavelength radar observations. J. Atmos. Sci., 57, 2417-2434.

— B. A. Albrecht, R. Lhermitte, and A. Savtchenko, 2001: Radar observations of updrafts, downdrafts, and turbulence in fair-weather cumuli. J. Atmos. Sci., 58, 1750-1766.

Korolev, A. V., 1995: The influence of supersaturation fluctuations on droplet size spectra formation. J. Atmos. Sci., 52, 3620-3634.

LeMone, M. A., 1973: The structure and dynamics of horizontal roll vortices in the planetary boundary layer. J. Atmos. Sci., 30, 1077-1091.

_, 1990: Some observations of vertical velocity skewness in the convective planetary boundary layer. J. Atmos. Sci., 47, $1163-1169$. 
Lenschow, D. H., J. C. Wyngaard, and W. T. Pennell, 1980: Meanfield and second-moment budgets in a baroclinic, convective boundary layer. J. Atmos. Sci., 37, 1313-1326.

Lothon, M., D. H. Lenschow, D. Leon, and G. Vali, 2005: Turbulence measurements in marine stratocumulus with airborne Doppler radar. Quart. J. Roy. Meteor. Soc., 131, 2063-2080.

Magaritz, L., M. Pinsky, and A. Khain, 2009: Investigation of droplet size distributions and drizzle formation using a new trajectory ensemble model. Part II: Lucky parcels. J. Atmos. Sci., 66, 781-805.

Moisseev, D. N., V. Chandrasekar, C. M. H. Unal, and H. W. J. Russchenberg, 2006: Dual-polarization spectral analysis for retrieval of effective raindrop shapes. J. Atmos. Oceanic Technol., 23, 1682-1695.

Monin, A. S., and A. M. Yaglom, 1975: Statistical Fluid Mechanics: Mechanics of Turbulence. Vol. 2, The MIT Press, 874 pp.

Muschinski, A., R. G. Frehlich, and B. B. Balsley, 2004: Small-scale and large-scale intermittency in the nocturnal boundary layer and the residual layer. J. Fluid Mech., 515, 319-351.

O'Connor, E. J., R. J. Hogan, and A. J. Illingworth, 2005: Retrieving stratocumulus drizzle parameters using Doppler radar and lidar. J. Appl. Meteor., 44, 14-27.

Orr, B. W., and R. A. Kropfli, 1999: A method for estimating particle fall velocities from vertically pointing Doppler radar. J. Atmos. Oceanic Technol., 16, 29-37.

Petters, M. D., J. R. Snider, B. Stevens, G. Vali, I. Faloona, and L. Russel, 2006: Accumulation mode aerosol, pockets of open cells, and particle nucleation in the remote subtropical
Pacific marine boundary layer. J. Geophys. Res., 111, D02206, doi:10.1029/2004JD005694.

Pinsky, M., L. Magaritz, A. Khain, O. Krasnov, and A. Sterkin, 2008: Investigation of droplet size distributions and drizzle formation using a new trajectory ensemble model. Part I: Model description and first results in a nonmixing limit. J. Atmos. Sci., 65, 2064-2086.

Shupe, M. D., P. Kollias, M. Poellot, and E. Eloranta, 2008: On deriving vertical air motions from cloud radar Doppler spectra. J. Atmos. Oceanic Technol., 25, 547-557.

Siebert, H., K. Lehmann, and M. Wendisch, 2006: Observations of small-scale turbulence and energy dissipation rates in the cloudy boundary layer. J. Atmos. Sci., 63, 1451-1466.

Stevens, B., and Coauthors, 2003: Dynamics and Chemistry of Maritime Stratocumulus-DYCOMS-II. Bull. Amer. Meteor. Soc., 84, 579-593.

_ , and Coauthors, 2005a: Evaluation of large-eddy simulations via observations of nocturnal marine stratocumulus. Mon. Wea. Rev., 133, 1443-1455.

- G. Vali, K. Comstock, R. Wood, M. C. vanZanten, P. H. Austin, C. S. Bretherton, and D. H. Lenshow, 2005b: Pockets of open cells (POCs) and drizzle in marine stratocumulus. Bull. Amer. Meteor. Soc., 86, 51-57.

Yanovsky, F., H. W. J. Russchenberg, and C. M. H. Unal, 2005: Retrieval of information about turbulence in rain by using Doppler polarimetric radar. IEEE Trans. Microw. Theory Tech., 53, 444-450. 